\title{
Floristic and phytosociological analysis of palm swamps in the central part of the Brazilian savanna ${ }^{1}$
}

\author{
Isa Lucia de Morais Resende ${ }^{2,5}$, Lázaro José Chaves ${ }^{3}$ and José Ângelo Rizzo ${ }^{4}$
}

Submitted: 5 March, 2011. Accepted: 28 December, 2012

\begin{abstract}
We analyzed the floristics and phytosociology of three palm swamps in the municipality of Bela Vista de Goiás, located in the state of Goiás, Brazil, in the central part of the Brazilian savanna (Cerrado). The floristic surveys were conducted monthly from May 2008 to April 2009, and 310 species were recorded (seven bryophytes, 15 ferns and 288 angiosperms). Bryophytes belonged to five genera and five families; ferns belonged to nine genera and nine families; and angiosperms belonged to 134 genera and 45 families. The angiosperm families with the highest species richness were Poaceae, Cyperaceae, Asteraceae, Eriocaulaceae, Xyridaceae, Lentibulariaceae, Melastomataceae, Rubiaceae and Fabaceae. The palm swamps were divided into three zones of increasing humidity: edge, middle and core. The number of species was higher in the middle than at the edge and the core. The families with the highest cover values were Cyperaceae, Melastomataceae, Arecaceae and Poaceae. Although the palm swamps had been disturbed to varying degrees, those disturbances did not affect the flora in the middle or the core. Floristic similarity was high between these two zones within a given palm swamp and low between the edges of different palm swamps.
\end{abstract}

Key words: wetlands; Brazilian savanna; plant diversity

\section{Introduction}

Wetlands are environments in which water is the determining factor of abiotic and biotic conditions (Keddy 2000, Steinke \& Saito 2008). These areas support several typical animal and plant species and foster a large number of ecological processes that sustain those species (Maltchik et al. 2003), making wetlands some of the most productive environments on the planet (Steinke \& Saito 2008).

In the Brazilian savanna (Cerrado), despite the dominance of phytophysiognomies on well-drained soils, wetlands such as palm swamps also occur (Meireles et al. 2002). Palm swamps are communities composed of a continuous herb-grass layer, which occupies most of their area, and a tree-shrub layer dominated by individuals of the palm Mauritia flexuosa L. f. (local name, buriti), with a canopy cover of between $5 \%$ and $10 \%$ (Ribeiro \& Walter 2008).

In addition to the diversity of plant species (Araújo et al. 2002, Guimarães et al. 2002), palm swamps present some distinct micro-ecosystems in which there are some plant-animal interactions that are still poorly understood (Oliveira et al. 2009). These environments serve as refuges, food sources and reproduction sites for the aquatic and terrestrial fauna of the adjacent phytophysiognomies (Guimarães et al. 2002). They are also sites of great scenic beauty with economic and sustainable potential for ecotourism and for extractivism involving species such as M. flexuosa and Syngonanthus nitens (Bong.) Ruhland (capim-dourado, which means "golden grass") by small rural communities (Schmidt et al. 2008).

Palm swamps are categorized as areas of permanent preservation, for ensuring the maintenance of springs and the quality of watercourses (Araújo et al. 2002). Although legally protected, palm swamps have suffered human disturbances that occasionally become irreversible, mainly because of their low regenerative capacity (Carvalho 1991, Araújo et al. 2002, Guimarães et al. 2002). Considering the importance and the condition of degradation of palm swamps, it is necessary to expand studies on the physical, biotic, social and anthropic aspects of this physiognomy, in a dynamic and multidisciplinary manner. Thus, it will be

\footnotetext{
${ }^{1}$ Based on the Doctoral thesis of the first Author.

${ }^{2}$ Universidade Estadual de Goiás, Unidade Universitária, Quirinópolis, GO, Brazil

${ }^{3}$ Universidade Federal de Goiás, Departamento de Melhoramento Genético de Plantas, Goiânia, GO, Brazil

${ }^{4}$ Universidade Federal de Goiás, Unidade de Conservação/PRPPG/Herbário, Goiânia, GO, Brazil

${ }^{5}$ Author for correspondence: isamorais1@gmail.com
} 
possible to understand the aspects that characterize palm swamps and to support measures to mitigate possible environmental impacts. There have been a few studies on palm swamp vegetation (Araújo et al. 2002; Guimarães et al. 2002; Ramos 2004; Oliveira et al. 2009). However, there have been no studies on the plant diversity of this physiognomy in the central part of the Cerrado. Therefore, the aim of this study was to contribute to the knowledge of the floristic composition of palm swamps in the central part of the Cerrado and to conduct a phytosociological survey in those swamps.

\section{Material and methods}

The study sites were three disturbed palm swamps, which were selected based on previous studies involving satellite images and field trips. All three sites are located on private properties in the city of Bela Vista de Goiás, in the state of Goiás, Brazil, and are part of the riparian vegetation of the tributaries of the Peixe River, which is in turn a tributary of the Corumbá River, pertaining to the Paranaíba River watershed (Fig. 1).

The palm swamp with the lowest degree of disturbance was in an area of transition to cerradão and was located upstream of dams for the provisioning of cattle. The $M$. flexuosa individuals at that study site displayed signs of burning on the stems and of trampling by cattle. The other two palm swamps did not display signs of burning, and, at the time of sampling, one was surrounded by cultivated pasture dominated by Urochloa spp., whereas the other was surrounded by crops (primarily corn and soybean). The palm swamp surrounded by crops was the only one that was not at least partially fenced off. This site suffered interference by cattle, which were released by cattle farmers in order to graze on crop residues (mainly of corn) during the postharvest period. We also observed signs of swine, which were allowed to feed at the site on a regular basis.

According to the Köppen classification system, the climate in the region is type Aw, which is defined as megathermal, with a rainy summer and a dry winter. Mean monthly temperatures range from $19^{\circ} \mathrm{C}$ to $28^{\circ} \mathrm{C}$ and mean annual rainfall is below $2000 \mathrm{~mm}$ (INMET 2009).

The floristic survey was conducted between May 2008 and April 2009, with monthly collection of fertile botanical material observed in asystematic walks throughout the three sites. Each palm swamp was subdivided into three zones: edge, middle and core (Araújo et al. 2002, Guimarães et al. 2002). The edge is closest to the transitional vegetation and exhibits soil that is lighter in color and more well-drained. The middle exhibits soil that is darker and periodically saturated with water. The core is permanently flooded. For all sampled material, we recorded data on the growth habit, zone of occurrence and morphological characteristics.

The botanical material was mounted following the usual procedures, and the vouchers were deposited at the Herbarium of the Federal University of Goiás. Species were iden- tified with the aid of specialists in various botanical families and by comparison with identified vouchers in herbaria. We adopted classification systems based on the following works: Goffinet \& Buck (2004) for Bryophyta; He-Nigrén et al. (2006) for Marchantiophyta; Tryon \& Tryon (1982) for Pteridophyta; and the Angiosperm Phylogeny Group 2009 update (APG III 2009) for Magnoliophyta (angiosperms).

The phytosociological survey was conducted with the line intercept method, adapted from Munhoz \& Felfili (2006), to determine the composition and linear cover of species. At each site, we selected four transects, $10 \mathrm{~m}$ apart, comprising two transects on each bank of the drainage canal. The transects were positioned perpendicular to the drainage line, and, for each transect, we extended a line from the edge to the core of the palm swamp. The line was divided in 1-m sections, or sample units. We analyzed 129 sample units in the palm swamp surrounded by cerradão, 246 in that surrounded by pasture and 115 in that surrounded by crops. For each 1-m section, we recorded the occurrence of each species and its horizontal projection. The sum of the horizontal projections of a given species in all sample units was taken as the absolute cover value for that species in each palm swamp. The relative cover value was obtained by dividing the absolute cover value of each species by the sum of the absolute cover values of all species, and multiplying the result by 100 . The record of occurrence of each species in the sample units was used in order to calculate the frequency of species in each palm swamp. To investigate the dynamics of the vegetation, the first phytosociological survey was performed in October 2009 (at the end of the dry season) and the second was performed in March 2010 (at the end of the rainy season), both surveys being performed in the same transects.

To evaluate the diversity of the community, we used an adaptation of the Shannon diversity index, using the cover values of both sampling periods instead of the number of individuals, in accordance with the method suggested by Munhoz \& Felfili (2006). The floristic similarity between the palm swamps and between the zones (edge, middle and core) of each site was evaluated by the quantitative Sørensen similarity index (Mueller-Dombois \& Ellenberg 1974). The data of floristic similarity (I) were transformed to dissimilarity indices $(\mathrm{D}=1-\mathrm{I})$ and evaluated with a cluster analysis using the unweighted pair group method with arithmetic mean, with the software GENES (Cruz, 2006).

\section{Results and discussion}

We sampled 310 species (Tab. 1): seven bryophytes, 15 ferns and 288 angiosperms. Bryophytes comprised five genera and five families; ferns comprised nine genera and nine families; and angiosperms comprised 134 genera, 45 families and one undetermined Poaceae, $64.9 \%$ of the genera and $42.2 \%$ of the families comprising only one species. The families with the largest number of species were Poaceae (n 


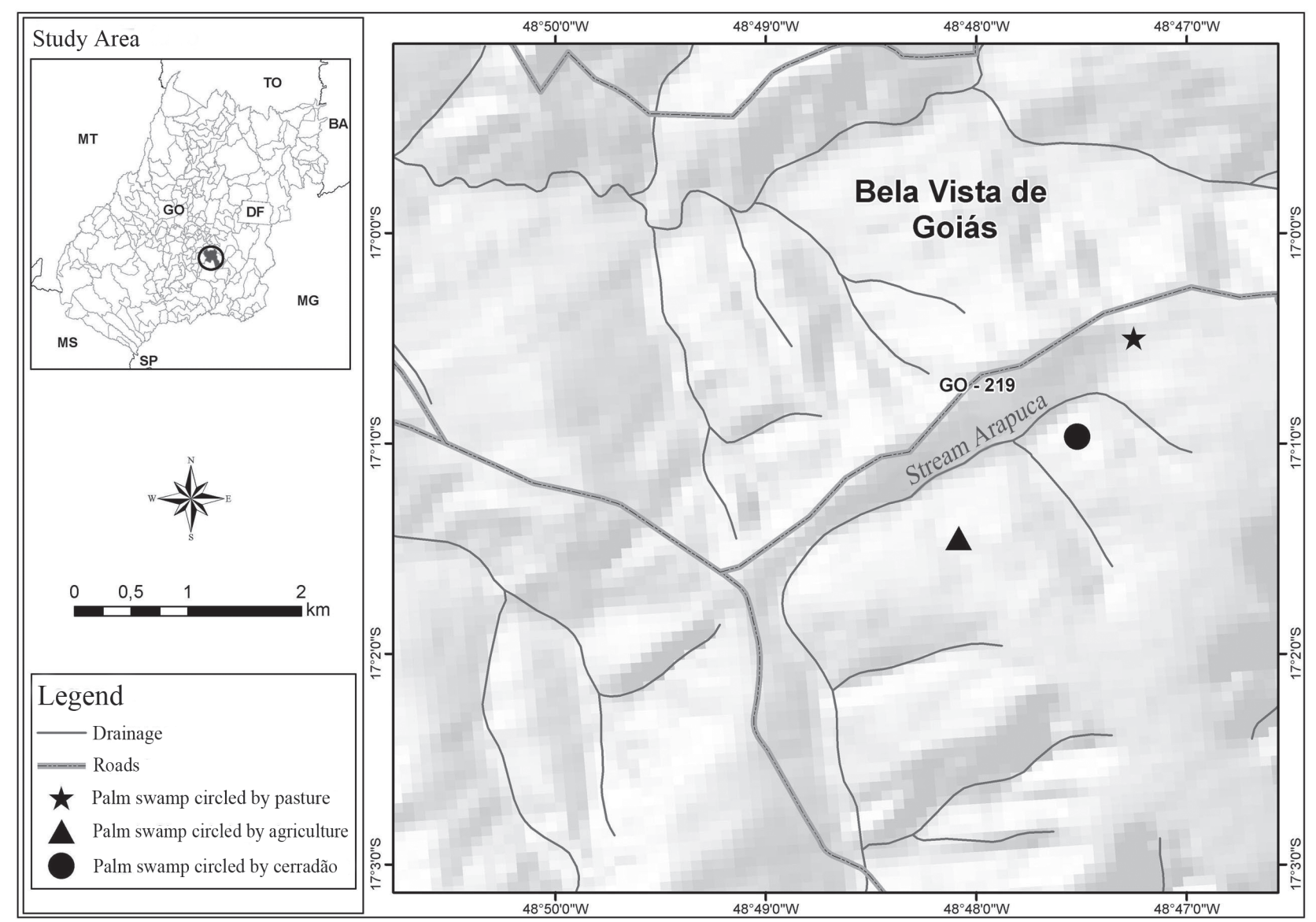

Figure 1. Location of the three palm swamp study sites in the city of Bela Vista de Goiás, state of Goiás, Brazil.

= 53), Cyperaceae $(\mathrm{n}=44)$, Asteraceae $(\mathrm{n}=24)$, Eriocaulaceae $(\mathrm{n}=18)$, Xyridaceae $(\mathrm{n}=17)$, Lentibulariaceae $(\mathrm{n}$ $=16)$, Melastomataceae $(\mathrm{n}=12)$, Rubiaceae $(\mathrm{n}=12)$ and Fabaceae $(\mathrm{n}=11)$, collectively accounting for $71.9 \%$ of the sampled angiosperms. In the studies conducted by Araújo et al. (2002), Guimarães et al. (2002), Ramos (2004), and Oliveira et al. (2009), these same families, with the exception of Lentibulariaceae, also showed the highest species richness. Guimarães et al. (2002) registered no Lentibulariaceae species and only three Xyridaceae species.

The number of species recorded was equal or comparable among the three sites: 187 in the palm swamp surrounded by pasture; 187 in the palm swamp surrounded by crops; and 186 in the palm swamp surrounded by cerradão. Floristic similarity between the sites was high: $58.2 \%$ between the palm swamp surrounded by pasture and that surrounded by crops; $61.9 \%$ between the palm swamp surrounded by pasture and that surrounded by cerradão; and $55.1 \%$ between the palm swamp surrounded by cerradão and that surrounded by crops. According to Kent \& Coker (1992), values $\geq 50 \%$ indicate high similarity. Although the sites had been disturbed to varying degrees, those disturbances did not affect the flora in the middle or core zones of these palm swamps, only the species at their edges.
Floristic similarity was higher between the middle and core zones and lower between the edge zones (Tab. 2). The dendrogram resulting from a cluster analysis shows the floristic similarity between the middle and the core within each palm swamp, as well as the dissimilarity between the sites (Fig. 2). The coefficient of cophenetic correlation was 0.70 , indicating good representativeness of the original dissimilarity indices in the dendrogram. There was floristic dissimilarity between the edges of the three palm swamps and between the edge and the other two zones within each palm swamp. Despite belonging to different sites, the three edge zones were more similar to each other than to their respective middle and core zones. This might be attributable to the fact that an edge is a zone of transition to the adjacent vegetation. In the palm swamp surrounded by pasture and the palm swamp surrounded by crops, the colonization by diaspores of exotic or invasive species, originating from the human activities in the vicinity, might have contributed to the differences between edge zones.

The Sørensen similarity index between the zones (edge, middle and core) within each palm swamp was higher between the middle and the core and lower between the edge and the core (Tab. 2). The highest floristic similarity (76.4\%) was between the middle and the core of the site surrounded 
Table 1. Species, ordered by category and family, sampled in the edge, middle and core zones (E, $M$ and C, respectively) of three palm swamps-one surrounded by pasture (PP); one surrounded by crops (PCr); and one surrounded by cerradão (PC)—in the city of Bela Vista de Goiás, state of Goiás, Brazil.

\begin{tabular}{|c|c|c|c|c|c|c|c|c|c|}
\hline \multirow{2}{*}{$\begin{array}{l}\text { CATEGORY } \\
\qquad \begin{array}{r}\text { FAMILY } \\
\text { Species }\end{array}\end{array}$} & \multicolumn{3}{|c|}{ PP } & \multicolumn{3}{|c|}{$\mathrm{PCr}$} & \multicolumn{3}{|c|}{ PC } \\
\hline & $\mathrm{E}$ & M & $\mathrm{C}$ & $\mathrm{E}$ & M & $\mathrm{C}$ & $\mathrm{E}$ & M & $\mathrm{C}$ \\
\hline \multicolumn{10}{|l|}{ BRYOPHYTES } \\
\hline \multicolumn{10}{|l|}{ ANEURACEAE } \\
\hline Riccardia cataractarum (Spruce) Schiffner & & $\mathrm{X}$ & & & & & & & \\
\hline Riccardia sinuata (Hook.) Trevis. & & & $\mathrm{x}$ & & & & & & \\
\hline \multicolumn{10}{|l|}{ BRACHYTHECIACEAE } \\
\hline Zelometeorium patulum (Hedw.) Manuel & & $\mathrm{X}$ & & & & $\mathrm{X}$ & & & \\
\hline \multicolumn{10}{|l|}{ DICRANACEAE } \\
\hline Leucoloma serrulatum Brid. ${ }^{* *}$ & & $\mathrm{X}$ & & & & & & & \\
\hline \multicolumn{10}{|l|}{ PYLAISIADELPHACEAE } \\
\hline Isopterygium tenerifolium Mitt. & & & & & & & & $\mathrm{X}$ & \\
\hline \multicolumn{10}{|l|}{ SPHAGNACEAE } \\
\hline Sphagnum cyclophyllum Sull. \& Lesq. & & $\mathrm{X}$ & $\mathrm{X}$ & & & & & & \\
\hline Sphagnum perichaetiale Hampe & & $\mathrm{X}$ & & & & & & & \\
\hline \multicolumn{10}{|l|}{ SEEDLESS VASCULAR PLANTS } \\
\hline \multicolumn{10}{|l|}{ BLECHNACEAE } \\
\hline Blechnum serrulatum Rich. North, Central and South American* & & $\mathrm{X}$ & & & & & & & \\
\hline \multicolumn{10}{|l|}{ CYATHEACEAE } \\
\hline Cyathea atrovirens (Langsd. \& Fisch.) Domin ${ }^{* * *}$ & & & $\mathrm{X}$ & & & $\mathrm{x}$ & & & $\mathrm{x}$ \\
\hline \multicolumn{10}{|l|}{ DENNSTAEDTIACEAE } \\
\hline Lindsaea lancea (L.) Bedd. Central and South American* & & $\mathrm{X}$ & & & & & & & \\
\hline \multicolumn{10}{|l|}{ GLEICHENIACEAE } \\
\hline Dicranopteris flexuosa (Schrad.) Underw. North, Central and South American* & & $\mathrm{X}$ & & & & $\mathrm{x}$ & & & \\
\hline \multicolumn{10}{|l|}{ HYMENOPHYLLACEAE } \\
\hline Trichomanes crispum L. South American ${ }^{*}$ & & $\mathrm{X}$ & & & & & & & \\
\hline \multicolumn{10}{|l|}{ LYCOPODIACEAE } \\
\hline Lycopodiella alopecuroides (L.) Cranfill. North, Central and South American* & & $\mathrm{X}$ & $\mathrm{X}$ & & & & & $\mathrm{X}$ & $\mathrm{X}$ \\
\hline Lycopodiella camporum B. Øllg. \& P.G. Windisch & $\mathrm{X}$ & $\mathrm{X}$ & $\mathrm{X}$ & & & & & $\mathrm{X}$ & $\mathrm{X}$ \\
\hline Lycopodiella caroliniana (L.) Pic. Serm. South American* & & & & & & & & $\mathrm{X}$ & $\mathrm{X}$ \\
\hline Lycopodiella cernua (L.) Pic. Serm. & & $\mathrm{x}$ & $\mathrm{X}$ & & $\mathrm{X}$ & $\mathrm{x}$ & & $\mathrm{X}$ & $\mathrm{X}$ \\
\hline \multicolumn{10}{|l|}{ OPHIOGLOSSACEAE } \\
\hline Ophioglossum crotalophoroides Walter neotropical ${ }^{*}$ - North American ${ }^{\star *}$ & & & & & & & & $\mathrm{X}$ & \\
\hline \multicolumn{10}{|l|}{ PTERIDACEAE } \\
\hline Pityrogramma calomelanos (L.) Link. neotropical ${ }^{*}$ & & $\mathrm{X}$ & & & $\mathrm{X}$ & $\mathrm{x}$ & & & $\mathrm{X}$ \\
\hline \multicolumn{10}{|l|}{ THELYPTERIDACEAE } \\
\hline Thelypteris biformata (Rosenst.) R.M. Tryon ${ }^{* * *}$ & & & & & & $\mathrm{X}$ & & & \\
\hline Thelypteris eriosora (Fée) Ponce ${ }^{* * *}$ & & & & & $\mathrm{X}$ & $\mathrm{X}$ & & $\mathrm{X}$ & $\mathrm{X}$ \\
\hline Thelypteris opposita (Vahl) Ching North, Central and South American* & & $\mathrm{X}$ & & & & & & & $\mathrm{X}$ \\
\hline Thelypteris serrata (Cav.) Alston & & $\mathrm{X}$ & $\mathrm{X}$ & & $\mathrm{X}$ & $\mathrm{X}$ & & $\mathrm{X}$ & $\mathrm{X}$ \\
\hline \multicolumn{10}{|l|}{ ANGIOSPERMS } \\
\hline \multicolumn{10}{|l|}{ ACANTHACEAE } \\
\hline Geissomeria pubescens Nees** & $\mathrm{X}$ & & & & & & & & \\
\hline Ruellia sp. & $\mathrm{X}$ & $\mathrm{X}$ & & & & & $X$ & & \\
\hline
\end{tabular}

Continues 
Table 1. Continuation.

\begin{tabular}{|c|c|c|c|c|c|c|c|c|c|}
\hline \multirow{2}{*}{$\begin{array}{l}\text { CATEGORY } \\
\qquad \begin{array}{r}\text { FAMILY } \\
\text { Species }\end{array}\end{array}$} & \multicolumn{3}{|c|}{$\mathrm{PP}$} & \multicolumn{3}{|c|}{$\mathrm{PCr}$} & \multicolumn{3}{|c|}{ PC } \\
\hline & E & M & $\mathrm{C}$ & $\mathrm{E}$ & M & $\mathrm{C}$ & $\mathrm{E}$ & M & $\mathrm{C}$ \\
\hline \multicolumn{10}{|l|}{ AMARANTHACEAE } \\
\hline Alternanthera brasiliana (L.) Kuntze & & & & $\mathrm{X}$ & & & & & \\
\hline \multicolumn{10}{|l|}{ ANACARDIACEAE } \\
\hline Tapirira guianensis Aubl. & & & $\mathrm{X}$ & & & & & & \\
\hline \multicolumn{10}{|l|}{ ANNONACEAE } \\
\hline Cardiopetalum calophyllum Schltdl. & $\mathrm{X}$ & & & & & & & & \\
\hline Xylopia aromatica (Lam.) Mart. & $\mathrm{x}$ & & & & & & & $\mathrm{X}$ & \\
\hline Xylopia emarginata Mart. & & $\mathrm{X}$ & $\mathrm{X}$ & & & $\mathrm{X}$ & & & \\
\hline \multicolumn{10}{|l|}{ APIACEAE } \\
\hline Eryngium ebracteatum Lam. Central and South American* & & & & $\mathrm{X}$ & $\mathrm{X}$ & & & $\mathrm{X}$ & $\mathrm{x}$ \\
\hline \multicolumn{10}{|l|}{ AQUIFOLIACEAE } \\
\hline Ilex affinis Gardner & $\mathrm{X}$ & $\mathrm{X}$ & $\mathrm{X}$ & & & $\mathrm{x}$ & & & \\
\hline \multicolumn{10}{|l|}{ ARACEAE } \\
\hline Urospatha sagittifolia (Rudge) Schott & & $\mathrm{X}$ & $\mathrm{X}$ & & $\mathrm{X}$ & $\mathrm{X}$ & & $\mathrm{X}$ & $\mathrm{X}$ \\
\hline Xanthosoma striatipes (Kunth \& C.D. Bouché) Madison & & $\mathrm{X}$ & & $\mathrm{X}$ & $\mathrm{X}$ & $\mathrm{X}$ & & $\mathrm{X}$ & $\mathrm{X}$ \\
\hline \multicolumn{10}{|l|}{ ARECACEAE } \\
\hline Mauritia flexuosa L.f. & & $\mathrm{X}$ & $\mathrm{X}$ & & $\mathrm{X}$ & $\mathrm{X}$ & & $\mathrm{X}$ & $\mathrm{X}$ \\
\hline \multicolumn{10}{|l|}{ ASTERACEAE } \\
\hline Acanthospermum australe (Loefl.) Kuntze neotropical ${ }^{\star}$ - South American? & & & & & & & $\mathrm{X}$ & & \\
\hline Achyrocline alata (Kunth) DC. South American* & $\mathrm{X}$ & $\mathrm{X}$ & & & $\mathrm{X}$ & & & & \\
\hline Achyrocline satureioides (Lam.) DC. South American* & $\mathrm{X}$ & $\mathrm{X}$ & & & $\mathrm{X}$ & & & & \\
\hline Adenostemma suffruticosum Gardner & & & & & $\mathrm{X}$ & & $\mathrm{X}$ & & \\
\hline Ageratum conyzoides L. pantropical ${ }^{*}$ - neotropical, western India & & & & $\mathrm{x}$ & $\mathrm{x}$ & & & $\mathrm{X}$ & \\
\hline Bidens pilosa L. neotropical ${ }^{*}-$ South American & $\mathrm{x}$ & & & & & & $\mathrm{X}$ & & \\
\hline Conyza bonariensis (L.) Cronquist neotropical ${ }^{*}$ & $\mathrm{X}$ & & & & & & & & \\
\hline Elephantopus mollis Kunth pantropical - neotropical & & & & & & & $\mathrm{X}$ & & \\
\hline Emilia fosbergii Nicolson pantropical ${ }^{*}$ - Bahamas?; old world? & $\mathrm{X}$ & & & $\mathrm{X}$ & $\mathrm{X}$ & & & & \\
\hline Emilia sonchifolia (L.) DC. old world ${ }^{*}$ - African? & $\mathrm{X}$ & & & & & & & & \\
\hline Erechtites hieraciifolius (Fisch. ex Spreng.) Griseb. South American* & $\mathrm{x}$ & $\mathrm{X}$ & & $\mathrm{X}$ & $\mathrm{X}$ & $\mathrm{X}$ & & $\mathrm{X}$ & $\mathrm{X}$ \\
\hline Eupatorium amygdalinum Lam. ${ }^{* * *}$ & & $\mathrm{X}$ & & & & & & & $\mathrm{X}$ \\
\hline Eupatorium tremulum Hook. \& Arn..** & & $\mathrm{X}$ & & & & $\mathrm{X}$ & & $\mathrm{X}$ & $\mathrm{X}$ \\
\hline Mikania officinalis Mart. & $\mathrm{x}$ & $\mathrm{X}$ & & & $\mathrm{X}$ & & $\mathrm{X}$ & $\mathrm{X}$ & \\
\hline Mikania psilostachya DC. South American* & & & & & & $\mathrm{X}$ & & & \\
\hline Porophyllum ruderale (Jacq.) Cass. & & & & $\mathrm{x}$ & & & $\mathrm{X}$ & & \\
\hline Praxelis clematidea (Griseb.) R.M. King \& H. Rob. & & & & $\mathrm{X}$ & & & & & \\
\hline Praxelis grandiflora (DC.) Sch. Bip. & $\mathrm{x}$ & & & $\mathrm{X}$ & & & $\mathrm{X}$ & & \\
\hline Praxelis kleinioides (Kunth) Sch. Bip. & & & & $\mathrm{X}$ & & & $\mathrm{X}$ & & \\
\hline Riencourtia oblongifolia Gardner & & & & & & & & $\mathrm{X}$ & $\mathrm{X}$ \\
\hline Vernonia ararana Gardner*** & & $\mathrm{X}$ & $\mathrm{X}$ & $\mathrm{X}$ & $\mathrm{X}$ & & $\mathrm{X}$ & $\mathrm{X}$ & $\mathrm{X}$ \\
\hline Vernonia helophila Mart. ex DC.*1 & $\mathrm{X}$ & & & $\mathrm{X}$ & & & & & \\
\hline Vernonia polyanthes Less. ${ }^{2}$ & $\mathrm{X}$ & & & & & & & & \\
\hline Vernonia rubriramea Mart. ex DC. & $\mathrm{X}$ & $\mathrm{X}$ & & & & $\mathrm{x}$ & & & \\
\hline \multicolumn{10}{|l|}{ BURMANNIACEAE } \\
\hline Burmannia capitata (Walter ex J.F. Gmel.) Mart. North, Central and South American* & & $\mathrm{X}$ & $\mathrm{X}$ & & $\mathrm{x}$ & & & & \\
\hline Burmannia damazii Beauverd & & $\mathrm{X}$ & $\mathrm{X}$ & & & & & $\mathrm{X}$ & $\mathrm{X}$ \\
\hline Burmannia flava Mart. North, Central and South American* & & $\mathrm{X}$ & $\mathrm{X}$ & & $\mathrm{X}$ & & & $\mathrm{X}$ & $\mathrm{X}$ \\
\hline Burmannia grandiflora Malme & & $\mathrm{X}$ & $\mathrm{X}$ & & $\mathrm{x}$ & & & $\mathrm{X}$ & $\mathrm{X}$ \\
\hline
\end{tabular}


Table 1. Continuation

\begin{tabular}{|c|c|c|c|c|c|c|c|c|c|}
\hline \multirow{2}{*}{$\begin{array}{l}\text { CATEGORY } \\
\qquad \begin{array}{r}\text { FAMILY } \\
\text { Species }\end{array}\end{array}$} & \multicolumn{3}{|c|}{ PP } & \multicolumn{3}{|c|}{$\mathrm{PCr}$} & \multicolumn{3}{|c|}{ PC } \\
\hline & E & M & $\mathrm{C}$ & $\mathrm{E}$ & M & $\mathrm{C}$ & E & M & $\mathrm{C}$ \\
\hline \multicolumn{10}{|l|}{ CHLORANTHACEAE } \\
\hline Hedyosmum brasiliense Miq. & & & & & & $\mathrm{X}$ & & & \\
\hline \multicolumn{10}{|l|}{ COMMELINACEAE } \\
\hline Commelina benghalensis L. paleotropical ${ }^{*}$ - India, southeast of Asia & & & & $\mathrm{X}$ & & & & & \\
\hline \multicolumn{10}{|l|}{ CYPERACEAE } \\
\hline Ascolepis brasiliensis (Kunth) Benth. ex C.B. Clarke pantropical ${ }^{*}$ - neotropical & & $\mathrm{X}$ & $\mathrm{X}$ & & $\mathrm{X}$ & $\mathrm{x}$ & & $\mathrm{X}$ & $\mathrm{X}$ \\
\hline Bulbostylis capillaris (L.) C.B. Clarke & & $\mathrm{X}$ & & & & & & & \\
\hline Bulbostylis sellowiana (Kunth) Palla & $\mathrm{X}$ & $\mathrm{X}$ & & & $\mathrm{X}$ & $\mathrm{X}$ & & $\mathrm{X}$ & $\mathrm{X}$ \\
\hline Calyptrocarya glomerulata (Brongn.) Urb. neotropical ${ }^{*}$ & & $\mathrm{X}$ & & & $\mathrm{X}$ & & & & \\
\hline Calyptrocarya luzuliformis T. Koyama ${ }^{* * 3}$ & & $\mathrm{X}$ & & & & & & & \\
\hline Cyperus ferax Rich. tropical cosmopolitan & & & & & $\mathrm{X}$ & & & & \\
\hline Cyperus haspan L. pantropical ${ }^{\star}$ & $\mathrm{X}$ & $\mathrm{X}$ & $\mathrm{X}$ & & $\mathrm{X}$ & $\mathrm{X}$ & & $\mathrm{X}$ & $\mathrm{X}$ \\
\hline Cyperus lanceolatus Poir. & & & & $\mathrm{X}$ & $\mathrm{X}$ & $\mathrm{x}$ & & $\mathrm{X}$ & $\mathrm{X}$ \\
\hline Cyperus luzulae (L.) Rottb. ex Retz. neotropical ${ }^{*}$ & & & & $\mathrm{X}$ & $\mathrm{X}$ & & & & \\
\hline Eleocharis acutangula (Roxb.) Schult. pantropical ${ }^{*}$ & & & & & & $\mathrm{X}$ & & & $\mathrm{X}$ \\
\hline Eleocharis capillacea Kunth Caribbean; Central and South American* & & $\mathrm{X}$ & $\mathrm{X}$ & $\mathrm{X}$ & $\mathrm{X}$ & $\mathrm{X}$ & & $\mathrm{X}$ & $\mathrm{X}$ \\
\hline Eleocharis filiculmis Kunth neotropical ${ }^{*}$ & & $\mathrm{X}$ & & & $\mathrm{x}$ & & & $\mathrm{X}$ & \\
\hline Eleocharis geniculata (L.) Roem. \& Schult. & & $\mathrm{X}$ & & $\mathrm{X}$ & $\mathrm{X}$ & $\mathrm{x}$ & & & \\
\hline Eleocharis nuda C.B. Clarke***** & & & & & & $\mathrm{X}$ & & $\mathrm{X}$ & $\mathrm{X}$ \\
\hline Eleocharis obtusetrigona (Lindl. \& Nees) Steud. & & & & $\mathrm{X}$ & & $\mathrm{X}$ & & & $\mathrm{X}$ \\
\hline Fimbristylis autumnalis (L.) Roem. \& Schult. neotropical ${ }^{*}$ & $\mathrm{X}$ & $\mathrm{X}$ & & & $\mathrm{X}$ & $\mathrm{X}$ & $\mathrm{X}$ & $\mathrm{X}$ & $\mathrm{X}$ \\
\hline Fimbristylis complanata (Retz.) Link pantropical ${ }^{\star}$ - neotropical & $\mathrm{X}$ & $\mathrm{X}$ & & & & & & & \\
\hline Fimbristylis dichotoma (L.) Vahl pantropical ${ }^{*}$ & & $\mathrm{X}$ & & $\mathrm{X}$ & $\mathrm{X}$ & & $\mathrm{X}$ & & \\
\hline Fimbristylis spadicea (L.) Vahl & & & & & & & $\mathrm{x}$ & & \\
\hline Kyllinga pumila Michx. neotropical and paleotropical ${ }^{*}$ & & & & $\mathrm{X}$ & $\mathrm{X}$ & & & & \\
\hline Lipocarpha humboldtiana Nees & $\mathrm{X}$ & $\mathrm{X}$ & $\mathrm{X}$ & & $\mathrm{X}$ & $\mathrm{X}$ & & $\mathrm{X}$ & $\mathrm{X}$ \\
\hline Pycreus capillifolius (A. Rich.) C.B. Clarke neotropical and paleotropical ${ }^{\star}$ ? & & & & $\mathrm{X}$ & $\mathrm{X}$ & & & & \\
\hline Pycreus megapotamicus (Kunth) Nees ${ }^{* *, * * *}$ & & & & & $\mathrm{x}$ & $\mathrm{x}$ & & & \\
\hline Rhynchospora brasiliensis Boeck. & & $\mathrm{X}$ & & & $\mathrm{X}$ & & & $\mathrm{X}$ & \\
\hline Rhynchospora consanguinea (Kunth) Boeck. & $\mathrm{x}$ & $\mathrm{X}$ & $\mathrm{X}$ & & $\mathrm{x}$ & & $\mathrm{X}$ & $\mathrm{X}$ & $\mathrm{X}$ \\
\hline Rhynchospora corymbosa (L.) Britton pantropical ${ }^{\star}$ - neotropical & & $\mathrm{X}$ & & & $\mathrm{X}$ & & & $\mathrm{X}$ & $\mathrm{X}$ \\
\hline Rhynchospora emaciata (Nees) Boeck. South American* & & & & & $\mathrm{x}$ & & & $\mathrm{X}$ & $\mathrm{X}$ \\
\hline Rhynchospora eximia (Nees) Boeck. neotropical ${ }^{*}$ & & $\mathrm{X}$ & & & $\mathrm{x}$ & & & & \\
\hline Rhynchospora globosa (Kunth) Roem. \& Schult. neotropical ${ }^{*}$ & $\mathrm{x}$ & $\mathrm{X}$ & $\mathrm{X}$ & & $\mathrm{x}$ & $\mathrm{x}$ & & $\mathrm{X}$ & $\mathrm{X}$ \\
\hline Rhynchospora hirsuta (Vahl) Vahl ${ }^{* * * * *}$ & & $\mathrm{X}$ & & & $\mathrm{X}$ & & & $\mathrm{X}$ & \\
\hline Rhynchospora marisculus Lindl. ex Nees Central and South American* & & & & & & & & $\mathrm{X}$ & \\
\hline Rhynchospora riparia (Nees) Boeckeler South American* & & & & & $\mathrm{X}$ & $\mathrm{X}$ & & & \\
\hline Rhynchospora robusta (Kunth) Boeck. & $\mathrm{X}$ & $\mathrm{X}$ & $\mathrm{X}$ & & $\mathrm{X}$ & $\mathrm{x}$ & $\mathrm{X}$ & $\mathrm{X}$ & $\mathrm{X}$ \\
\hline Rhynchospora rugosa (Vahl) Gale neotropical ${ }^{\star}$ & & & & & & & $\mathrm{X}$ & $\mathrm{X}$ & \\
\hline Rhynchospora speciosa (Kunth) Boeck. & $\mathrm{X}$ & $\mathrm{X}$ & & & & & $\mathrm{X}$ & $\mathrm{X}$ & \\
\hline Rhynchospora tenerrima Nees ex Sprengel Central and South American* & & & & $\mathrm{X}$ & $\mathrm{X}$ & & & & \\
\hline Rhynchospora tenuis Willd. ex Link neotropical ${ }^{*}$ & & $\mathrm{X}$ & $\mathrm{X}$ & & $\mathrm{X}$ & $\mathrm{X}$ & & $\mathrm{x}$ & $\mathrm{X}$ \\
\hline
\end{tabular}


Table 1. Continuation.

\begin{tabular}{|c|c|c|c|c|c|c|c|c|c|}
\hline \multirow{2}{*}{$\begin{array}{l}\text { CATEGORY } \\
\qquad \begin{array}{r}\text { FAMILY } \\
\text { Species }\end{array}\end{array}$} & \multicolumn{3}{|c|}{$\mathrm{PP}$} & \multicolumn{3}{|c|}{$\mathrm{PCr}$} & \multicolumn{3}{|c|}{$\mathrm{PC}$} \\
\hline & $\mathrm{E}$ & M & $\mathrm{C}$ & $\mathrm{E}$ & M & $\mathrm{C}$ & $\mathrm{E}$ & $\mathrm{M}$ & $\mathrm{C}$ \\
\hline \multicolumn{10}{|l|}{ CYPERACEAE } \\
\hline Rhynchospora velutina (Kunth) Boeck. neotropical ${ }^{*}$ & $\mathrm{X}$ & $\mathrm{X}$ & & & $\mathrm{X}$ & & & $\mathrm{X}$ & \\
\hline Scleria clarkei Lindm.***** & & & & & $\mathrm{X}$ & & & & \\
\hline Scleria distans Poir. pantropical ${ }^{*}$ & $\mathrm{X}$ & $\mathrm{X}$ & & & $\mathrm{X}$ & & & & \\
\hline Scleria hirta Boeck..*,*** & & $\mathrm{X}$ & $\mathrm{X}$ & & & & & $\mathrm{X}$ & $\mathrm{X}$ \\
\hline Scleria hirtella Sw. pantropical ${ }^{\star}$ - Caribbean & $\mathrm{X}$ & $\mathrm{X}$ & & & & & & $\mathrm{x}$ & $\mathrm{X}$ \\
\hline Scleria reticularis Michx. neotropical ${ }^{*}$ & & & & & $\mathrm{X}$ & & & $\mathrm{X}$ & $\mathrm{X}$ \\
\hline Scleria retroserrata Kük.**,*** & & & & & $\mathrm{X}$ & & & & \\
\hline \multicolumn{10}{|l|}{ DILLENIACEAE } \\
\hline Curatella americana L. Caribbean, Central and South American* & & & & & $\mathrm{X}$ & & & $\mathrm{X}$ & \\
\hline Davilla nitida (Vahl) Kubitzki & & & $\mathrm{X}$ & & $\mathrm{X}$ & & & & \\
\hline \multicolumn{10}{|l|}{ DROSERACEAE } \\
\hline Drosera communis A. St.-Hil. & & $\mathrm{X}$ & $\mathrm{X}$ & & $\mathrm{X}$ & $\mathrm{X}$ & & $\mathrm{X}$ & $\mathrm{X}$ \\
\hline \multicolumn{10}{|l|}{ ERIOCAULACEAE } \\
\hline Eriocaulon crassiscapum Bong. & & & & & $\mathrm{X}$ & $\mathrm{X}$ & & & \\
\hline Eriocaulon humboldtii Kunth & & $\mathrm{X}$ & $\mathrm{X}$ & & $\mathrm{X}$ & $\mathrm{x}$ & & $\mathrm{X}$ & $\mathrm{X}$ \\
\hline Eriocaulon kunthii Körn..$^{* * * * *}$ & & & & & & & & $\mathrm{X}$ & $\mathrm{X}$ \\
\hline Eriocaulon linearifolium Körn. & & $\mathrm{X}$ & $\mathrm{X}$ & & $\mathrm{X}$ & $\mathrm{X}$ & & $\mathrm{X}$ & $\mathrm{X}$ \\
\hline Eriocaulon modestum Kunth & $\mathrm{X}$ & & & & $\mathrm{x}$ & $\mathrm{x}$ & & & \\
\hline Eriocaulon steyermarkii Moldenke & & $\mathrm{X}$ & $\mathrm{X}$ & & & & & $\mathrm{X}$ & $\mathrm{X}$ \\
\hline Leiothrix angustifolia Ruhland & & $\mathrm{X}$ & $\mathrm{X}$ & & & & & & \\
\hline Leiothrix schlechtendalii Ruhland & & $\mathrm{x}$ & $\mathrm{X}$ & & & & & & \\
\hline Paepalanthus flaccidus Kunth & & $\mathrm{x}$ & $\mathrm{X}$ & & & $\mathrm{X}$ & & & $\mathrm{x}$ \\
\hline Syngonanthus anthemidiflorus (Bong.) Ruhland & & $\mathrm{x}$ & $\mathrm{x}$ & & & & & & \\
\hline Syngonanthus appressus (Körn.) Ruhland & $\mathrm{X}$ & $\mathrm{x}$ & $\mathrm{x}$ & & & & & $\mathrm{X}$ & $\mathrm{X}$ \\
\hline Syngonanthus caulescens (Poir.) Ruhland & & $\mathrm{X}$ & $\mathrm{X}$ & & $\mathrm{X}$ & $\mathrm{X}$ & & $\mathrm{X}$ & $\mathrm{X}$ \\
\hline Syngonanthus densiflorus (Körn.) Ruhland & & $\mathrm{X}$ & $\mathrm{x}$ & & $\mathrm{x}$ & $\mathrm{x}$ & & $\mathrm{x}$ & $\mathrm{X}$ \\
\hline Syngonanthus fuscescens Ruhland & & & & & & & & $\mathrm{x}$ & $\mathrm{X}$ \\
\hline Syngonanthus goyazensis Ruhland & & & & & $\mathrm{X}$ & $\mathrm{X}$ & & & $\mathrm{X}$ \\
\hline Syngonanthus gracilis (Bong.) Ruhland & & $\mathrm{X}$ & $\mathrm{X}$ & & & $\mathrm{X}$ & & $\mathrm{X}$ & $\mathrm{X}$ \\
\hline Syngonanthus nitens (Bong.) Ruhland & & $\mathrm{X}$ & $\mathrm{X}$ & & $\mathrm{X}$ & $\mathrm{x}$ & & $\mathrm{X}$ & $\mathrm{X}$ \\
\hline Syngonanthus schwackei Ruhland $d^{3 * *}$ & & & & & & & & $\mathrm{X}$ & $\mathrm{X}$ \\
\hline \multicolumn{10}{|l|}{ EUPHORBIACEAE } \\
\hline Chamaesyce potentilloides (Boiss.) Croizat South American* & & & & & & & $\mathrm{x}$ & $\mathrm{X}$ & $\mathrm{X}$ \\
\hline Croton glandulosus L. North, Central and South American* & $\mathrm{x}$ & $\mathrm{x}$ & & $\mathrm{x}$ & & & $\mathrm{X}$ & $\mathrm{X}$ & \\
\hline Croton sclerocalyx (Didr.) Müll. Arg. & $\mathrm{X}$ & $\mathrm{X}$ & & $\mathrm{X}$ & & & $\mathrm{X}$ & & \\
\hline \multicolumn{10}{|l|}{ FABACEAE } \\
\hline Aeschynomene paniculata Willd. ex Vogel North, Central and South American* & & & & $\mathrm{X}$ & & & $\mathrm{X}$ & $\mathrm{X}$ & \\
\hline Camptosema coriaceum (Nees \& Mart.) Benth. & $\mathrm{X}$ & & & & & & & & \\
\hline Chamaecrista diphylla (L.) Greene Caribbean; North, Central and South American ${ }^{\star}$ & & & & & & & $\mathrm{X}$ & & \\
\hline Chamaecrista trichopoda (Benth.) Britton \& Rose ex Britton \& Killip & & & & & & & $\mathrm{x}$ & & \\
\hline Crotalaria velutina Benth. & & & & $\mathrm{x}$ & & & & & \\
\hline Desmodium barbatum (L.) Benth. pantropical ${ }^{*}$ & $\mathrm{X}$ & $\mathrm{X}$ & & $\mathrm{x}$ & $\mathrm{X}$ & & $\mathrm{X}$ & $\mathrm{X}$ & \\
\hline Desmodium incanum DC. pantropical ${ }^{*}$ & $\mathrm{x}$ & $\mathrm{x}$ & & $\mathrm{X}$ & & & & & \\
\hline Mimosa foliolosa Benth. & & & & $\mathrm{X}$ & & & & & \\
\hline Stylosanthes nunoi Brandão & & & & & & & $\mathrm{X}$ & & \\
\hline Stylosanthes viscosa (L.) Sw. & & & & & & & $\mathrm{x}$ & & \\
\hline Zornia latifolia Sm. & & & & $\mathrm{X}$ & & & $\mathrm{X}$ & & \\
\hline
\end{tabular}


Table 1. Continuation.

\begin{tabular}{|c|c|c|c|c|c|c|c|c|c|}
\hline \multirow{2}{*}{$\begin{array}{l}\text { CATEGORY } \\
\qquad \begin{array}{r}\text { FAMILY } \\
\text { Species }\end{array}\end{array}$} & \multicolumn{3}{|c|}{ PP } & \multicolumn{3}{|c|}{$\mathrm{PCr}$} & \multicolumn{3}{|c|}{ PC } \\
\hline & $\mathrm{E}$ & M & $\mathrm{C}$ & $\mathrm{E}$ & M & $\mathrm{C}$ & $\mathrm{E}$ & M & $\mathrm{C}$ \\
\hline \multicolumn{10}{|l|}{ GENTIANACEAE } \\
\hline Curtia tenuifolia (Aubl.) Knobl. & & $\mathrm{X}$ & $\mathrm{X}$ & & & & & $\mathrm{X}$ & $\mathrm{X}$ \\
\hline Helia oblongifolia Mart. ${ }^{4 * *}$ & & $\mathrm{X}$ & & & $\mathrm{X}$ & & & & \\
\hline Irlbachia alata (Aubl.) Maas neotropical ${ }^{*}$ & & $\mathrm{X}$ & $\mathrm{X}$ & $\mathrm{X}$ & $\mathrm{X}$ & $\mathrm{x}$ & $\mathrm{x}$ & $\mathrm{X}$ & $\mathrm{X}$ \\
\hline Schultesia aptera Cham. & & $\mathrm{x}$ & $\mathrm{X}$ & & $\mathrm{X}$ & & $\mathrm{X}$ & $\mathrm{X}$ & $\mathrm{X}$ \\
\hline Schultesia gracilis Mart. & & $\mathrm{X}$ & $\mathrm{X}$ & & & & & & \\
\hline Tetrapollinia caerulescens (Aubl.) Maguire \& B.M. Boom ${ }^{4 * *}$ & & $\mathrm{X}$ & & & & & & & \\
\hline \multicolumn{10}{|l|}{ GESNERIACEAE } \\
\hline Sinningia elatior (Kunth) Chautems & $\mathrm{X}$ & $\mathrm{X}$ & & & & & & $\mathrm{X}$ & \\
\hline \multicolumn{10}{|l|}{ IRIDACEAE } \\
\hline Cipura paludosa Aubl. & & & & & & & $\mathrm{x}$ & & \\
\hline Sisyrinchium vaginatum Spreng. neotropical ${ }^{*}$ & & & & & & & $\mathrm{X}$ & $\mathrm{X}$ & $\mathrm{X}$ \\
\hline \multicolumn{10}{|l|}{ LAMIACEAE (LABIATAE) } \\
\hline Hypenia macrantha (A. St.-Hil. ex Benth.) Harley & $\mathrm{X}$ & & & & & & & & \\
\hline Hyptis caespitosa A. St.-Hil. ex Benth. & & & & $\mathrm{X}$ & $\mathrm{X}$ & & & & \\
\hline Hyptis carpinifolia Benth. & & & & & & & & $\mathrm{X}$ & $\mathrm{X}$ \\
\hline Hyptis lantanifolia Poit.neotropical ${ }^{*}$ & $\mathrm{X}$ & $\mathrm{X}$ & & $\mathrm{X}$ & & & & $\mathrm{X}$ & \\
\hline Hyptis subrotunda Pohl ex Benth. & $\mathrm{X}$ & & & & & & & & \\
\hline Hyptis tenuifolia Epling & $\mathrm{X}$ & $\mathrm{X}$ & & & & & $\mathrm{x}$ & $\mathrm{X}$ & \\
\hline Hyptis velutina Pohl ex Benth. & $\mathrm{X}$ & $\mathrm{x}$ & & & & & & & \\
\hline Marsypianthes chamaedrys (Vahl) Kuntze Central and South American* & $\mathrm{X}$ & & & $\mathrm{X}$ & & & & & \\
\hline \multicolumn{10}{|l|}{ LAURACEAE } \\
\hline Ocotea spixiana (Nees) Mez & & & & & & $\mathrm{x}$ & & & \\
\hline \multicolumn{10}{|l|}{ LENTIBULARIACEAE } \\
\hline Genlisea filiformis A. St.-Hil. Central and South American* & & & & & & & & $\mathrm{X}$ & $\mathrm{X}$ \\
\hline Genlisea pygmaea A. St.-Hil. South American* & & & & & & & & $\mathrm{X}$ & $\mathrm{X}$ \\
\hline Genlisea repens Benj. South American* & & & & & & & & $\mathrm{X}$ & $\mathrm{X}$ \\
\hline Utricularia amethystina Salzm. ex A. St.-Hil. \& Girard & & $\mathrm{x}$ & $\mathrm{X}$ & & $\mathrm{X}$ & $\mathrm{x}$ & & & \\
\hline Utricularia cucullata A. St.-Hil. \& Girard & & $\mathrm{X}$ & $\mathrm{X}$ & & & & & $\mathrm{X}$ & $\mathrm{X}$ \\
\hline Utricularia gibba L. pantropical ${ }^{*}$ & & & & & & & & $\mathrm{X}$ & $\mathrm{X}$ \\
\hline Utricularia huntii P. Taylor & & $\mathrm{x}$ & $\mathrm{X}$ & & & & & & \\
\hline Utricularia hispida Lam. & $\mathrm{X}$ & $\mathrm{X}$ & $\mathrm{X}$ & & $\mathrm{X}$ & $\mathrm{x}$ & & $\mathrm{X}$ & $\mathrm{X}$ \\
\hline Utricularia nana A. St.-Hil. \& Girard & & & $\mathrm{X}$ & & & & & $\mathrm{X}$ & $\mathrm{X}$ \\
\hline Utricularia nervosa Weber ex Benj. ${ }^{4 * *}$ & & $\mathrm{X}$ & $\mathrm{X}$ & & & & & $\mathrm{X}$ & $\mathrm{X}$ \\
\hline Utricularia nigrescens Sylvén & & $\mathrm{X}$ & $\mathrm{X}$ & & $\mathrm{X}$ & $\mathrm{x}$ & & $\mathrm{X}$ & $\mathrm{X}$ \\
\hline Utricularia praelonga A.St.-Hil. \& Girard & & $\mathrm{X}$ & $\mathrm{X}$ & & $\mathrm{X}$ & $\mathrm{x}$ & & $\mathrm{X}$ & $\mathrm{X}$ \\
\hline Utricularia pusilla Vahl & & $\mathrm{X}$ & $\mathrm{X}$ & & & & & $\mathrm{X}$ & $\mathrm{x}$ \\
\hline Utricularia trichophylla Spruce ex Oliv. & & $\mathrm{X}$ & $\mathrm{X}$ & & $\mathrm{X}$ & $\mathrm{x}$ & & $\mathrm{X}$ & $\mathrm{x}$ \\
\hline Utricularia triloba Benj. South American* & & $\mathrm{X}$ & $\mathrm{X}$ & & & & $\mathrm{x}$ & $\mathrm{X}$ & $\mathrm{X}$ \\
\hline Utricularia sp. & & $\mathrm{X}$ & $\mathrm{X}$ & & & & & $\mathrm{X}$ & $\mathrm{X}$ \\
\hline \multicolumn{10}{|l|}{ LIMNOCHARITACEAE } \\
\hline Limnocharis flava (L.) Buchenau & & & & & & & & & $\mathrm{X}$ \\
\hline
\end{tabular}


Table 1. Continuation.

\begin{tabular}{|c|c|c|c|c|c|c|c|c|c|}
\hline \multirow{2}{*}{$\begin{array}{r}\text { CATEGORY } \\
\text { FAMILY } \\
\text { Species } \\
\end{array}$} & \multicolumn{3}{|c|}{$\mathrm{PP}$} & \multicolumn{3}{|c|}{$\mathrm{PCr}$} & \multicolumn{3}{|c|}{$\mathrm{PC}$} \\
\hline & $\mathrm{E}$ & M & $\mathrm{C}$ & $\mathrm{E}$ & M & $\mathrm{C}$ & $\mathrm{E}$ & M & $\mathrm{C}$ \\
\hline \multicolumn{10}{|l|}{ LYTHRACEAE } \\
\hline Cuphea carthagenensis (Jacq.) J.F. Macbr. Central and South American* & & & & $\mathrm{X}$ & & & & & \\
\hline Cuphea micrantha Kunth & & & & $\mathrm{X}$ & & & $\mathrm{X}$ & $\mathrm{X}$ & \\
\hline Cuphea polymorpha A. St.-Hil. & & $\mathrm{X}$ & & & & & & & \\
\hline Diplusodon lanceolatus Pohl & & & & & & & $\mathrm{X}$ & $\mathrm{X}$ & \\
\hline \multicolumn{10}{|l|}{ MALPIGHIACEAE } \\
\hline Banisteriopsis pubipetala (A. Juss.) Cuatrec. & & $\mathrm{X}$ & & & & & & & \\
\hline Camarea affinis A. St.-Hil. & & & & $\mathrm{X}$ & & & $\mathrm{X}$ & & \\
\hline \multicolumn{10}{|l|}{ MALVACEAE } \\
\hline Corchorus hirtus L. North, Central and South American* & & & & $\mathrm{X}$ & & & & & \\
\hline Melochia sp. & & & & $\mathrm{X}$ & & & & & \\
\hline Sida linifolia Cav. pantropical ${ }^{\star}$ - South American? & $\mathrm{X}$ & $\mathrm{X}$ & & $\mathrm{X}$ & $\mathrm{X}$ & & & & \\
\hline Waltheria tomentosa $\mathrm{H}$. St. John & $\mathrm{X}$ & & & $\mathrm{X}$ & $\mathrm{X}$ & & $\mathrm{X}$ & $\mathrm{X}$ & \\
\hline \multicolumn{10}{|l|}{ MELASTOMATACEAE } \\
\hline Desmoscelis villosa (Aubl.) Naudin & & $\mathrm{X}$ & & & $\mathrm{X}$ & $\mathrm{x}$ & & & \\
\hline Macairea radula (Bonpl.) DC. & & $\mathrm{X}$ & & & $\mathrm{X}$ & & & & \\
\hline Miconia albicans (Sw.) Steud. Central and South American* & $\mathrm{X}$ & & & & & & $\mathrm{X}$ & & \\
\hline Miconia chamissois Naudin & & $\mathrm{X}$ & $\mathrm{X}$ & & $\mathrm{X}$ & $\mathrm{x}$ & & $\mathrm{X}$ & $\mathrm{X}$ \\
\hline Miconia elegans Cogn. & $\mathrm{X}$ & $\mathrm{X}$ & & & & & & & $\mathrm{X}$ \\
\hline Miconia ibaguensis (Bonpl.) Triana Central and South American* & & & & & & $\mathrm{x}$ & & & \\
\hline Miconia stenostachya DC. Central and South American* & $\mathrm{X}$ & & & & & & $\mathrm{X}$ & & \\
\hline Microlicia euphorbioides Mart. & & $\mathrm{X}$ & & & $\mathrm{X}$ & & & $\mathrm{X}$ & \\
\hline Microlicia helvola Triana & $\mathrm{X}$ & $\mathrm{X}$ & & & & & & $\mathrm{x}$ & \\
\hline Rhynchanthera grandiflora (Aubl.) DC. & $\mathrm{X}$ & $\mathrm{X}$ & & & $\mathrm{X}$ & & $\mathrm{X}$ & $\mathrm{X}$ & $\mathrm{X}$ \\
\hline Tibouchina gracilis (Bonpl.) Cogn. South American ${ }^{*}$ & & $\mathrm{x}$ & & & $\mathrm{X}$ & & & & \\
\hline Trembleya phlogiformis DC. & & $\mathrm{X}$ & $\mathrm{X}$ & & & & & $\mathrm{X}$ & $\mathrm{X}$ \\
\hline \multicolumn{10}{|l|}{ MELIACEAE } \\
\hline Guarea macrophylla Vahl South American* & & & $\mathrm{X}$ & & & & & & \\
\hline \multicolumn{10}{|l|}{ MYRTACEAE } \\
\hline Myrcia laruotteana Cambess. & $\mathrm{x}$ & & & & & & & & \\
\hline \multicolumn{10}{|l|}{ OCHNACEAE } \\
\hline Sauvagesia erecta L. pantropical ${ }^{*}$ & $\mathrm{X}$ & $\mathrm{X}$ & & & & & & & \\
\hline Sauvagesia racemosa A. St.-Hil. & $\mathrm{X}$ & $\mathrm{x}$ & $\mathrm{X}$ & & $\mathrm{X}$ & $\mathrm{x}$ & $\mathrm{X}$ & $\mathrm{X}$ & $\mathrm{X}$ \\
\hline Sauvagesia sp. & & $\mathrm{x}$ & & & $\mathrm{X}$ & & & & \\
\hline \multicolumn{10}{|l|}{ ONAGRACEAE } \\
\hline Ludwigia nervosa (Poir.) H. Hara & $\mathrm{X}$ & $\mathrm{X}$ & $\mathrm{x}$ & & $\mathrm{X}$ & $\mathrm{x}$ & $\mathrm{x}$ & $\mathrm{x}$ & $\mathrm{X}$ \\
\hline \multicolumn{10}{|l|}{ OROBANCHACEAE } \\
\hline Buchnera longifolia Kunth & $\mathrm{X}$ & $\mathrm{x}$ & & & & & & $\mathrm{x}$ & $\mathrm{X}$ \\
\hline \multicolumn{10}{|l|}{ PIPERACEAE } \\
\hline Piper aduncum L. neotropical ${ }^{*}$ & & & & & $\mathrm{X}$ & $\mathrm{x}$ & & & \\
\hline Piper flavicans C. DC. & & & & & & & & & $\mathrm{X}$ \\
\hline Piper fuligineum Kunth South American* & & & & & $\mathrm{X}$ & $\mathrm{x}$ & & $\mathrm{X}$ & $\mathrm{X}$ \\
\hline Piper sp. ${ }^{1}$ & & & & & $\mathrm{x}$ & $\mathrm{x}$ & & $\mathrm{x}$ & $\mathrm{X}$ \\
\hline Piper sp. ${ }^{2}$ & & & & & & & & & $\mathrm{X}$ \\
\hline
\end{tabular}


Table 1. Continuation.

\begin{tabular}{|c|c|c|c|c|c|c|c|c|c|}
\hline \multirow{2}{*}{$\begin{array}{l}\text { CATEGORY } \\
\qquad \begin{array}{r}\text { FAMILY } \\
\text { Species }\end{array}\end{array}$} & \multicolumn{3}{|c|}{$\mathrm{PP}$} & \multicolumn{3}{|c|}{$\mathrm{PCr}$} & \multicolumn{3}{|c|}{ PC } \\
\hline & E & M & $\mathrm{C}$ & E & M & $\mathrm{C}$ & E & $\mathrm{M}$ & $\mathrm{C}$ \\
\hline \multicolumn{10}{|l|}{ PLANTAGINACEAE } \\
\hline Scoparia dulcis L. pantropical ${ }^{*}$ & $\mathrm{X}$ & $\mathrm{X}$ & & $\mathrm{X}$ & & & & & \\
\hline \multicolumn{10}{|l|}{ PHYLLANTHACEAE } \\
\hline Hyeronima alchorneoides Allemão & & & & & & $\mathrm{X}$ & & & \\
\hline \multicolumn{10}{|l|}{ POACEAE } \\
\hline Andropogon bicornis L. Caribbean; North, Central and South American* & $\mathrm{X}$ & $\mathrm{X}$ & & $\mathrm{X}$ & $\mathrm{X}$ & & $\mathrm{X}$ & & \\
\hline Andropogon fastigiatus Sw. Central and South American* & & & & & $\mathrm{X}$ & & & & \\
\hline Andropogon lateralis Nees South American* & & & & $\mathrm{X}$ & $\mathrm{x}$ & & & & \\
\hline Andropogon leucostachyus Kunth Caribbean; North, Central and South American* & & $\mathrm{X}$ & & & $\mathrm{X}$ & & & $\mathrm{x}$ & \\
\hline Andropogon virgatus Desv. ex Ham. Central and South American* & $\mathrm{X}$ & $\mathrm{X}$ & & & $\mathrm{X}$ & & $\mathrm{X}$ & $\mathrm{X}$ & \\
\hline Andropogon sp. ${ }^{1}$ & & $\mathrm{X}$ & & $\mathrm{X}$ & $\mathrm{X}$ & & & $\mathrm{X}$ & \\
\hline Andropogon sp. ${ }^{2}$ & & $\mathrm{X}$ & & & $\mathrm{X}$ & & $\mathrm{X}$ & $\mathrm{X}$ & \\
\hline Andropogon sp. ${ }^{3}$ & & $\mathrm{X}$ & & & & & & & \\
\hline Andropogon sp. ${ }^{4}$ & & & & & $\mathrm{X}$ & & & & \\
\hline Andropogon sp. ${ }^{5}$ & & $\mathrm{X}$ & & $\mathrm{X}$ & $\mathrm{X}$ & & & $\mathrm{X}$ & \\
\hline Andropogon sp. ${ }^{6}$ & & $\mathrm{x}$ & & $\mathrm{x}$ & $\mathrm{x}$ & & & $\mathrm{x}$ & \\
\hline Andropogon sp. ${ }^{7}$ & & & & & & & & $\mathrm{X}$ & \\
\hline Anthaenantia lanata (Kunth) Benth. Central and South American* & & $\mathrm{X}$ & & & $\mathrm{x}$ & & & $\mathrm{X}$ & $\mathrm{X}$ \\
\hline Arthropogon villosus Nees & & & & & & & $\mathrm{X}$ & $\mathrm{X}$ & \\
\hline Arundinella hispida (Humb. \& Bonpl. ex Willd.) Kuntze & & & & & $\mathrm{X}$ & & & & \\
\hline Axonopus brasiliensis (Spreng.) Kuhlm. South American* & & $\mathrm{X}$ & & & & & & $\mathrm{x}$ & \\
\hline Axonopus comans (Trin. ex Döll) Kuhlm. & & $\mathrm{X}$ & & & & & & $\mathrm{x}$ & \\
\hline Axonopus sp. & & & & $\mathrm{X}$ & & & & $\mathrm{x}$ & \\
\hline Ctenium sp. & & & $\mathrm{X}$ & & & & & & \\
\hline Digitaria sp. & & & & $\mathrm{x}$ & & & & & \\
\hline Echinolaena inflexa (Poir.) Chase & $\mathrm{x}$ & $\mathrm{X}$ & $\mathrm{X}$ & & & & $\mathrm{X}$ & $\mathrm{X}$ & \\
\hline Elionurus muticus (Spreng.) Kybtze South American* & & & & $\mathrm{X}$ & & & & & \\
\hline Eragrostis secundiflora J. Presl North, Central and South American* & & & & & $\mathrm{x}$ & & & $\mathrm{X}$ & \\
\hline Eragrostis sp. ${ }^{1}$ & & & & $\mathrm{X}$ & & & & & \\
\hline Eragrostis sp. ${ }^{2}$ & & & & $\mathrm{X}$ & $\mathrm{X}$ & & & & \\
\hline Eragrostis sp. ${ }^{3}$ & & & & $\mathrm{X}$ & & & & & \\
\hline Eragrostis sp. ${ }^{4}$ & & & & $\mathrm{X}$ & & & & & \\
\hline Eriochrysis cayennensis P. Beauv. North, Central and South American* & $\mathrm{X}$ & $\mathrm{X}$ & $\mathrm{X}$ & & $\mathrm{X}$ & $\mathrm{X}$ & $\mathrm{X}$ & $\mathrm{X}$ & \\
\hline Eriochrysis holcoides (Nees) Kuhlm. South American* & & & & $\mathrm{X}$ & & & & & \\
\hline Hyparrhenia bracteata (Humb. \& Bonpl. ex Willd.) Stapf neotropical ${ }^{*}$ & $\mathrm{X}$ & $\mathrm{X}$ & & $\mathrm{X}$ & $\mathrm{X}$ & & $\mathrm{X}$ & $\mathrm{X}$ & $\mathrm{X}$ \\
\hline Hyparrhenia rufa (Nees) Stapf Caribbean; North, Central and South American* & & & & & & & & $\mathrm{X}$ & \\
\hline Ichnanthus procurrens (Nees ex Trin.) Swallen South American* & & & & $\mathrm{X}$ & $\mathrm{X}$ & & $\mathrm{X}$ & $\mathrm{X}$ & \\
\hline Luziola bahiensis (Steud.) Hitchc. North, Central and South American* & & & & & & & & & $\mathrm{x}$ \\
\hline Melinis minutiflora P. Beauv. African* & & $\mathrm{X}$ & & & $\mathrm{X}$ & & & & \\
\hline Otachyrium seminudum Hack. ex Send. \& Soderstr. & & $\mathrm{X}$ & $\mathrm{X}$ & & $\mathrm{X}$ & $\mathrm{X}$ & & $\mathrm{x}$ & $\mathrm{X}$ \\
\hline Panicum laxum Sw. & $\mathrm{X}$ & $\mathrm{X}$ & & $\mathrm{X}$ & $\mathrm{x}$ & & & $\mathrm{x}$ & \\
\hline Panicum parvifolium Lam. Caribbean; North, Central and South American* & & $\mathrm{X}$ & $\mathrm{X}$ & $\mathrm{X}$ & $\mathrm{X}$ & $\mathrm{X}$ & & $\mathrm{X}$ & $\mathrm{X}$ \\
\hline Paspalum compressifolium Swallen***** & & & & $\mathrm{X}$ & & & & & \\
\hline Paspalum imbricatum Filg. & & $\mathrm{X}$ & & & & & & & \\
\hline Paspalum maculosum Trin. Caribbean and South American* & & $\mathrm{X}$ & & & $\mathrm{x}$ & & & $\mathrm{X}$ & \\
\hline Paspalum multicaule Poir. Caribbean; Central and South American* & & & & $\mathrm{X}$ & & & & & \\
\hline Paspalum pilosum Lam. Caribbean; Central and South American & & $\mathrm{X}$ & & $\mathrm{X}$ & & & & & \\
\hline Paspalum plicatulum Michx. neotropical ${ }^{\star}$ & & & & $\mathrm{X}$ & & & & & \\
\hline
\end{tabular}


Table 1. Continuation.

\begin{tabular}{|c|c|c|c|c|c|c|c|c|c|}
\hline \multirow{2}{*}{$\begin{array}{l}\text { CATEGORY } \\
\qquad \begin{array}{r}\text { FAMILY } \\
\text { Species }\end{array}\end{array}$} & \multicolumn{3}{|c|}{$\mathrm{PP}$} & \multicolumn{3}{|c|}{$\mathrm{PCr}$} & \multicolumn{3}{|c|}{$\mathrm{PC}$} \\
\hline & E & M & $\mathrm{C}$ & E & M & $\mathrm{C}$ & $\mathrm{E}$ & M & $\mathrm{C}$ \\
\hline Paspalum stellatum Humb. \& Bonpl. ex Flüggé Caribbean; North, Central and South American* & & & & & & & & $\mathrm{X}$ & \\
\hline Pennisetum polystachion (L.) Schult. African* & $\mathrm{X}$ & & & $\mathrm{X}$ & & & & & \\
\hline Rhynchelytrum repens (Willd.) C.E. Hubb. & & & & $\mathrm{x}$ & & & & & \\
\hline Saccharum angustifolium (Nees) Trin. & & & $\mathrm{X}$ & & $\mathrm{X}$ & $\mathrm{X}$ & & $\mathrm{X}$ & $\mathrm{X}$ \\
\hline Saccharum asperum (Nees) Steud. South American* & & $\mathrm{X}$ & & & $\mathrm{x}$ & & & $\mathrm{X}$ & $\mathrm{X}$ \\
\hline Schizachyrium tenerum Nees North, Central and South American* & & $\mathrm{X}$ & & & & & & & \\
\hline Setaria parviflora (Poir.) Kerguélen North, Central and South American* & $\mathrm{X}$ & & & $\mathrm{X}$ & & & $\mathrm{X}$ & & \\
\hline Urochloa decumbens (Stapf) R.D. Webster African* & $\mathrm{X}$ & & & $\mathrm{x}$ & & & & & \\
\hline Urochloa humidicola (Rendle) Morrone \& Zuloaga African* & & & & $\mathrm{x}$ & & & & & \\
\hline Undetermined & & & & & $\mathrm{x}$ & & & $\mathrm{X}$ & \\
\hline \multicolumn{10}{|l|}{ POLYGALACEAE } \\
\hline Polygala galioides Poir. & & & & $\mathrm{x}$ & & & & & \\
\hline Polygala glochidata Kunth & & & & $\mathrm{x}$ & & & & & \\
\hline Polygala leptocaulis Torr. \& A. Gray North, Central and South American* & & & & & & & & $\mathrm{X}$ & $\mathrm{X}$ \\
\hline Polygala longicaulis Kunth & & & & & & & $\mathrm{X}$ & & \\
\hline Polygala nudicaulis Bennett,A.W. & & & & $\mathrm{X}$ & & & & & \\
\hline Polygala paniculata L. North, Central and South American* & $\mathrm{X}$ & $\mathrm{X}$ & & & $\mathrm{X}$ & & & $\mathrm{X}$ & $\mathrm{X}$ \\
\hline Polygala tenuis DC. & & & & & & & & $\mathrm{X}$ & $\mathrm{X}$ \\
\hline \multicolumn{10}{|l|}{ PRIMULACEAE } \\
\hline Lysimachia pumila Sw. American* - Caribbean? & & & & $\mathrm{X}$ & & & & & \\
\hline Rapanea intermedia $\mathrm{Mez}$ & & & & & & & $\mathrm{X}$ & & \\
\hline \multicolumn{10}{|l|}{ RUBIACEAE } \\
\hline Alibertia edulis (Rich.) A. Rich. ex DC. & $\mathrm{X}$ & & & & & $\mathrm{X}$ & $\mathrm{X}$ & $\mathrm{X}$ & \\
\hline Diodia teres Walter South American* & & & & $\mathrm{X}$ & & & & & \\
\hline Galianthe grandiflora E.L. Cabral & & & $\mathrm{X}$ & & & & & & \\
\hline Mitracarpus frigidus var. salzmannianus (DC.) K. Schum. South American* & & & & $\mathrm{X}$ & & & & & \\
\hline Mitracarpus hirtus (L.) DC. & & & & $\mathrm{x}$ & & & & & \\
\hline Psychotria carthagenensis Jacq. & & & $\mathrm{X}$ & & & & & & \\
\hline Psychotria paracatuensis Standl. & & & $\mathrm{x}$ & & $\mathrm{X}$ & $\mathrm{X}$ & & & \\
\hline Sipanea hispida Benth. ex Wernham & $\mathrm{X}$ & $\mathrm{X}$ & & $\mathrm{x}$ & $\mathrm{x}$ & & $\mathrm{X}$ & $\mathrm{X}$ & \\
\hline Spermacoce multiflora (DC.) Delprete ${ }^{5}$ & & $\mathrm{X}$ & & & $\mathrm{X}$ & & & & \\
\hline Spermacoce ovalifolia (M. Martens \& Galeotti) Hemsl. ${ }^{6}$ & & & & $\mathrm{X}$ & & & & & \\
\hline Spermacoce pulchristipula (Bremek.) Delprete ${ }^{7}$ & & & & & & & $\mathrm{X}$ & & \\
\hline Spermacoce schumannii (Standl. ex Bacigalupo) Delprete & & & $\mathrm{x}$ & $\mathrm{X}$ & $\mathrm{X}$ & $\mathrm{X}$ & $\mathrm{X}$ & $\mathrm{X}$ & $\mathrm{X}$ \\
\hline \multicolumn{10}{|l|}{ SANTALACEAE } \\
\hline Phoradendron affine (Pohl ex DC.) Engl. \& K. Krause & & & & & & $\mathrm{X}$ & & & \\
\hline Phoradendron crassifolium (Pohl ex DC.) Eichler & & $\mathrm{X}$ & & & & & & & \\
\hline Phoradendron mucronatum (DC.) Krug \& Urb. Caribbean and South American* & & & & & & & & $\mathrm{X}$ & $\mathrm{X}$ \\
\hline \multicolumn{10}{|l|}{ URTICACEAE } \\
\hline Cecropia pachystachya Trécul & & $\mathrm{X}$ & $\mathrm{x}$ & & $\mathrm{X}$ & $\mathrm{X}$ & & $\mathrm{X}$ & $\mathrm{X}$ \\
\hline \multicolumn{10}{|l|}{ VERBENACEAE } \\
\hline Stachytarpheta cayennensis (Rich.) Vahl & & & & & & & $\mathrm{X}$ & & \\
\hline \multicolumn{10}{|l|}{ XYRIDACEAE } \\
\hline Abolboda poarchon Seub. & & $\mathrm{X}$ & $\mathrm{X}$ & & & & & $\mathrm{X}$ & $\mathrm{X}$ \\
\hline Xyris aurea L.B. Sm. \& Downs & & $\mathrm{X}$ & $\mathrm{X}$ & & & & $\mathrm{X}$ & $\mathrm{X}$ & $\mathrm{X}$ \\
\hline Xyris jupicai Rich. North, Central and South American* & & $\mathrm{x}$ & $\mathrm{x}$ & & $\mathrm{X}$ & $\mathrm{X}$ & & $\mathrm{X}$ & $\mathrm{X}$ \\
\hline
\end{tabular}


Table 1. Continuation

\begin{tabular}{|c|c|c|c|c|c|c|c|c|c|c|}
\hline \multirow{2}{*}{$\begin{array}{l}\text { CATEGORY } \\
\text { FAMILY } \\
\text { Species }\end{array}$} & & \multicolumn{3}{|c|}{$\mathrm{PP}$} & \multicolumn{3}{|c|}{$\mathrm{PCr}$} & \multicolumn{3}{|c|}{ PC } \\
\hline & & E & M & $\mathrm{C}$ & $\mathrm{E}$ & M & $\mathrm{C}$ & E & M & $\mathrm{C}$ \\
\hline Xyris laxifolia Mart. South American* & & & $\mathrm{X}$ & $\mathrm{X}$ & & $\mathrm{x}$ & $\mathrm{x}$ & & $\mathrm{X}$ & $\mathrm{X}$ \\
\hline Xyris lutescens Kral \& Wand. & & & & & & & & & $\mathrm{X}$ & $\mathrm{X}$ \\
\hline Xyris savanensis Miq. South American* & & & $\mathrm{X}$ & $\mathrm{X}$ & & $\mathrm{x}$ & $\mathrm{x}$ & & $\mathrm{X}$ & $\mathrm{x}$ \\
\hline Xyris schizachne Mart. South American* & & & & & & & & & $\mathrm{X}$ & $\mathrm{X}$ \\
\hline Xyris tenella Kunth South American* & & & $\mathrm{X}$ & $\mathrm{X}$ & & & & & & \\
\hline Xyris tortula Mart. South American* & & & & & & & & & $\mathrm{x}$ & $\mathrm{X}$ \\
\hline Xyris sp. ${ }^{1}$ & & & $\mathrm{X}$ & $\mathrm{X}$ & & & & $\mathrm{X}$ & $\mathrm{X}$ & $\mathrm{X}$ \\
\hline Xyris sp. ${ }^{2}$ & & & & & & & & & $\mathrm{X}$ & $\mathrm{X}$ \\
\hline Xyris sp. ${ }^{3}$ & & & & & & $\mathrm{X}$ & $\mathrm{x}$ & & & \\
\hline Xyris sp. ${ }^{4}$ & & & & & & & & $\mathrm{x}$ & $\mathrm{x}$ & $\mathrm{X}$ \\
\hline Xyris sp. ${ }^{5}$ & & & $\mathrm{X}$ & $\mathrm{X}$ & & $\mathrm{X}$ & $\mathrm{X}$ & & $\mathrm{X}$ & $\mathrm{X}$ \\
\hline Xyris sp. ${ }^{6}$ & & & & $\mathrm{X}$ & & & & & $\mathrm{X}$ & $\mathrm{X}$ \\
\hline Xyris sp. ${ }^{7}$ & & $\mathrm{X}$ & $\mathrm{X}$ & $\mathrm{X}$ & & & $\mathrm{X}$ & & $\mathrm{X}$ & $\mathrm{X}$ \\
\hline Xyris sp. ${ }^{8}$ & & & $\mathrm{X}$ & $\mathrm{X}$ & & & & & $\mathrm{X}$ & $\mathrm{X}$ \\
\hline & Total species & 71 & 153 & 83 & 78 & 122 & 76 & 61 & 147 & 114 \\
\hline
\end{tabular}

${ }^{*}$ Species that were observed at the study sites but have a wide geographical distribution (neotropical, pantropical, cosmopolitan, etc.), for which the origin, or initial distribution, cannot be indicated in the Cerrado, including ruderal, invasive and exotic species, according to Mendonça et al. (2008).

${ }^{* *}$ New record for the state of Goiás.

${ }^{* * *}$ New record for the Cerrado, according to Mendonça et al. (2008).

${ }^{1}$ Vernonia helophila - although not cited by Mendonça et al. (2008), this species occurs in the states of Mato Grosso, São Paulo and Minas Gerais (Almeida 2008).

${ }^{2}$ Vernonia polyanthes - although not cited by Mendonça et al. (2008), this species occurs in the states of Goiás, Mato Grosso, Paraná, Bahia, São Paulo and Minas Gerais, and is considered a common species in the Cerrado (Almeida 2008).

${ }^{3}$ although not cited by Mendonça et al. (2008), this species has been sampled in the municipality of Jalapão, state of Tocantins (Rezende 2007).

${ }^{4}$ species previously sampled in palm swamps in the Triângulo Mineiro area, state of Minas Gerais (Oliveira et al. 2009).

${ }^{5}$ species previously sampled in the Federal District and in the state of Goiás (Delprete 2007).

${ }^{6}$ species previously sampled in the state of Goiás (Souza 2009).

${ }^{7}$ species with a wide distribution in South America (Delprete 2007).

by cerradão. The higher similarity between the middle and the core can be explained by the increase in humidity from the edge toward the core within a palm swamp, and by the fact that the middle and the core are less disturbed (Guimarães et al. 2002; Araújo et al. 2002). In contrast, edges are transitional zones to the adjacent phytophysiognomy and are more vulnerable to disturbances (such as cattle grazing and trampling, in palm swamps surrounded by pasture; Guimarães et al. 2002; Araújo et al. 2002) and invasion by exotic species (Oliveira et al. 2009). Sousa (2009) studied the soil texture in the three palm swamps evaluated in the present study and reported high sand content in the core zone, whereas the soil in the middle and at the edge had a high clay content, without significant textural differences. Therefore, we might infer that the topography is more important than is the soil texture in determining the establishment of humidity zones and the occurrence and floristic distribution of palm swamps, as Ramos et al. (2006) previously demonstrated in a study on the characterization of soils of palm swamps on different geomorphological surfaces in the Triângulo Mineiro region (state of Minas Gerais). From the dry edge toward the wet core within a palm swamp, there is a gradient of soil declivity and depth of the water table (Ramos et al. 2006; Guimarães et al. 2002), which alters the floristic composition of the three zones (Araújo et al. 2002).

Of the 310 species sampled, 79 (25\%) were common to all three sites, 53 were exclusive to the palm swamp surrounded by crops, 45 were exclusive to the palm swamp surrounded by cerradão, and 38 were exclusive to the palm swamp surrounded by pasture. Species exclusive to the site surrounded by crops included Alternanthera brasiliana (Amaranthaceae); Praxelis clematidea (Asteraceae); Commelina benghalensis (Commelinaceae); Cyperus ferax, C. luzulae and Pycreus capillifolius (Cyperaceae); Crotalaria velutina and Mimosa foliolosa (Fabaceae); Cuphea carthagenensis (Lythraceae); Corchorus hirtus and Melochia sp. 
(Malvaceae); Lysimachia pumila (Primulaceae); Digitaria sp.; four of the five Eragrostis species; and Urochloa humidicola (Poaceae). These were located mainly at the edge and might be considered invasive species. The diaspores of these plants originated from the surrounding crops. Species exclusive to the site surrounded by pasture included five of the seven bryophytes recorded in this study, as well as Geissomeria pubescens (Acanthaceae); Cardiopetalum calophyllum (Annonaceae); Conyza bonariensis, Emilia sonchifolia and Vernonia polyanthes (Asteraceae); Bulbostylis capillaris, Calyptrocarya luzuliformis and Fimbristylis complanata (Cyperaceae); both Leiothrix species and Syngonanthus anthemidiflorus (Eriocaulaceae); Camptosema coriaceum (Fabaceae); Tetrapollinia caerulescens (Gentianaceae); Hyptis subrotunda and $H$. velutina (Lamiaceae); Utricularia huntii (Lentibulariaceae); Ctenium sp., Paspalum imbricatum and Schizachyrium tenerum (Poaceae); and Xyris tenella (Xyridaceae). Despite the presence of invasive species, usually located at the edge, the species exclusive to this site were more characteristic of palm swamps and wetlands, such as bryophytes. This might have resulted from the fact that this site is fenced, which avoiding grazing and trampling by the cattle from the surrounding pasture.

Species that occurred only at the site surrounded by cerrado included Lycopodiella caroliniana (Lycopodiaceae); Ophioglossum crotalophoroides (Ophioglossaceae); Acanthospermum australe, Elephantopus mollis and Riencourtia oblongifolia (Asteraceae); Fimbristylis spadicea and Rhynchospora marisculus (Cyperaceae); Eriocaulon kunthii, Syngonanthus fuscescens and S. schwackei (Eriocaulaceae); two Chamaecrista species and two Stylosanthes species (Fabaceae), both of the Iridaceae species found in this study, the three Genlisea species and Utricularia gibba (Lentibulariaceae); Diplusodon lanceolatus (Lythraceae); Anthaenantia lanata, Luziola bahiensis and Paspalum stellatum (Poaceae); and five Xyris species (Xyridaceae). The palm swamp surrounded by cerradão and that surrounded by pasture displayed higher diversity of small, frail species, such as those of the families Lentibulariaceae, Burmanniaceae, Eriocaulaceae and Droseraceae. Conversely, in the palm swamp surrounded by crops, which was exposed to trampling by cattle and swine during the postharvest period, individuals of those families were rare or absent.

The fern Ophioglossum crotalophoroides (Ophioglossaceae), observed only in the palm swamp surrounded by cerrado, was not recorded in Goiás in the recent literature, and is considered a critically endangered species in Minas Gerais (COPAM 2008). Until now, it was sampled only in the Pantanal of the state of Mato Grosso do Sul (Cardoso et al. 2000), in the Federal District (Pereira et al. 2004; Felfili et al. 2007) and in the Quadrilátero Ferrífero area of Minas Gerais (Salino \& Almeida 2008). Regarding the bryophytes, this study is the first record of Leucoloma serrulatum (Dicranaceae) and the second record of Sphag- num cyclophyllum (Sphagnaceae) in Goiás (see Sousa et al. 2010; Yano 2011).

For all three study sites, the number of species was higher in the middle than in the other zones. However, for the palm swamp surrounded by cerradão, the difference between the middle and the core was small in comparison with that observed for the other two sites (Tab. 1). At the three sites, only Praxelis grandiflora (Asteraceae) and Setaria parviflora (Poaceae) were exclusive to the edge, and Cyathea atrovirens (Cyatheaceae) were exclusive to the core. In the middle and core of the three palm swamps, we identified the species Drosera communis (Droseraceae); Eriocaulon humboldtii, Syngonanthus caulescens, S. densiflorus and S. nitens (Eriocaulaceae); Utricularia nigrescens, U. praelonga and U. trichophylla (Lentibulariaceae); Miconia chamissois (Melastomataceae); Otachyrium seminudum (Poaceae); Cecropia pachystachya (Urticaceae); and Xyris jupicai, X. laxifolia, X. savanensis, and Xyris sp. (Xyridaceae). In the edge and middle of the three sites, the species Desmodium barbatum (Fabaceae) and Sipanea hispida (Rubiaceae) occurred. The distribution patterns of the remaining species differed among the three sites.

The Shannon diversity index was 2.8 for the palm swamp surrounded by pasture, 3.0 for the palm swamp surrounded by crops and 3.4 for the palm swamp surrounded by cerradão. In the few studies on the vegetation of palm swamps, this index has not been calculated. Therefore, we cannot yet determine whether our results are representative of these physiognomies, i.e., whether they can be classified as conserved or disturbed.

In the phytosociological surveys, we sampled 130 species belonging to 80 genera and 37 families (Tab. 3). In the two sampling periods, we recorded 86 species at the site surrounded by pasture, 81 at the site surrounded by crops and 96 at the site surrounded by cerradão. The families with the highest cover values in October 2009 and March 2010, respectively, were as follows: Cyperaceae (37.9\% and $21.4 \%)$, Melastomataceae (21.0\% and 20.6\%), Arecaceae (16.8\% and $18.0 \%)$, and Poaceae (11.1\% and 6.2\%) in the palm swamp surrounded by pasture; Melastomataceae (25.6\% and 37.9\%), Cyperaceae (20.7\% and $11.7 \%)$, Poaceae (17.5\% and 19.7\%), and Arecaceae (10.3\% and 16.3\%) in the palm swamp surrounded by crops; and Poaceae (29.3\% and 26.3\%), Cyperaceae (25.8\% and 25.8\%), Melastomataceae (18.0\% and $11.8 \%$ ), and Arecaceae (3.3\% and $11.0 \%$ ) in the palm swamp surrounded by cerradão (Fig. 3). For most of the families, relative frequency was higher than was relative cover, at all three study sites (Fig. 3). This might be attributable to the fact that most palm swamp species are herbs or subshrubs, small in height and diameter, and are widely distributed through the area (Munhoz \& Felfili 2006).

The families for which the relative cover was higher than was the relative frequency included Melastomataceae, Arecaceae, Cyperaceae, Poaceae and Urticaceae. The 
Table 2. Matrix of floristic similarity (Sørensen similarity index) between the nine zones of the palm swamps studied in the city of Bela Vista de Goiás, state of Goiás, Brazil.

\begin{tabular}{|c|c|c|c|c|c|c|c|c|c|}
\hline \multirow[b]{2}{*}{ Zone } & \multicolumn{9}{|c|}{ Zone } \\
\hline & EC & $\mathrm{MC}$ & $\mathrm{CC}$ & EP & MP & $\mathrm{CP}$ & ECr & $\mathrm{MCr}$ & $\mathrm{CCr}$ \\
\hline EC & 100.0 & 32.0 & 18.3 & 39.7 & 25.4 & 7.0 & 29.0 & 23.2 & 8.0 \\
\hline MC & & 100.0 & 76.4 & 31.5 & 61.7 & 16.4 & 21.5 & 57.9 & 21.6 \\
\hline CC & & & 100.0 & 14.0 & 23.5 & 20.3 & 8.3 & 26.8 & 38.6 \\
\hline EP & & & & 100.0 & 53.6 & 18.2 & 26.8 & 29.2 & 14.4 \\
\hline MP & & & & & 100.0 & 59.4 & 19.9 & 59.1 & 23.8 \\
\hline CP & & & & & & 100.0 & 1.9 & 13.6 & 30.3 \\
\hline $\mathrm{ECr}$ & & & & & & & 100.0 & 30.8 & 11.7 \\
\hline $\mathrm{MCr}$ & & & & & & & & 100.0 & 55.3 \\
\hline $\mathrm{CCr}$ & & & & & & & & & 100.0 \\
\hline
\end{tabular}

Prefixes: E - edge; $\mathrm{M}$ - middle; $\mathrm{C}$ - core.

Suffixes: C - palm swamp surrounded by cerrado; $\mathrm{P}$ - palm swamp surrounded by pasture; $\mathrm{Cr}$ - palm swamp surrounded by crops.

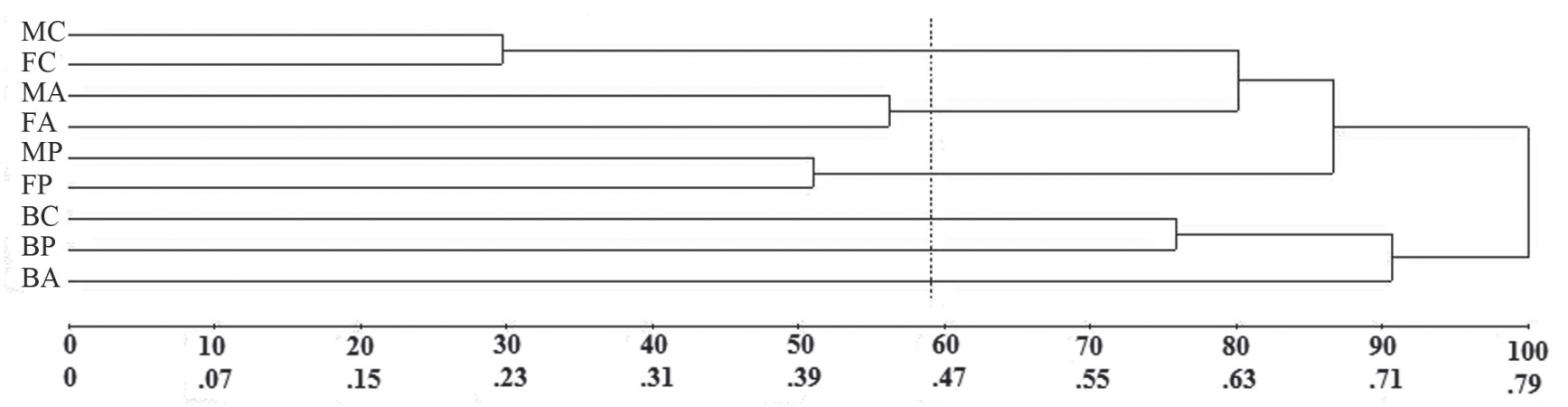

Figure 2. Dendrogram generated by the complement of the Sørensen similarity index ( $(\mathrm{D}=1-\mathrm{I})$ between the nine zones of the palm swamps studied in the city of Bela Vista de Goiás, state of Goiás, Brazil. Prefixes: E - edge; M - middle; C - core. Suffixes: C - palm swamp surrounded by cerradão; P - palm swamp surrounded by pasture; $\mathrm{Cr}$ - palm swamp surrounded by crops. Cophenetic correlation $=0.6982$.

species with the highest relative cover were Rhynchospora consanguinea, $R$. tenuis, $R$. globosa, $R$. robusta, Mauritia flexuosa, Miconia chamissois, Andropogon virgatus, A. bicornis, Rhynchanthera grandiflora and Saccharum angustifolium. These species were among the ten main species in terms of the cover values, in both sampling periods; there was only one change in order from one period to the next. In addition to these species, the following species were also among the ten species with the highest relative cover: Urospatha sagittifolia, Xyris sp., Psychotria paracatuensis, Syngonanthus densiflorus, S. caulescens and Ilex affinis in the palm swamp surrounded by pasture; Cecropia pachystachya, Hyptis lantanifolia, Xyris laxifolia and Piper aduncum in the palm swamp surrounded by crops; and Ludwigia nervosa, Xanthosoma striatipes, Andropogon sp. and Syngonanthus densiflorus in the palm swamp surrounded by cerradão. Species of Poaceae and Cyperaceae usually form dense clumps or have individuals that possess rhizomes or stolons and are widely distributed in the vegetation.
In contrast, the Melastomataceae species are shrubs and Arecaceae species are trees, as are Urticaceae species. This explains why relative cover was higher than was the relative frequency for those species.

There was a small difference between the two sampling periods in terms of the number of species. The number of species recorded in October 2009 and in March 2010 was, respectively, 60 and 74 in the palm swamp surrounded by cerradão, 65 and 72 in the palm swamp surrounded by pasture, and 61 and 57 in the palm swamp surrounded by crops. As can be seen, at the first two sites, there was a slight increase from October to March. This small variation in species number might have occurred because palm swamps have a seasonal area and a perennial area, the soil in the latter remaining water-saturated for several months of the year (Ramos 2004, Oliveira 2005).

At the study sites, the families with the highest species richness were Poaceae, Cyperaceae, Asteraceae, Eriocaulaceae, Xyridaceae, Lentibulariaceae, Melastomataceae, Ru- 
Table 3. Relative frequency $(\mathrm{RF})$ and relative cover $(\mathrm{RC})$ of the species sampled in three palm swamps-one surrounded by pasture (PP); one surrounded by crops (PCr); and one surrounded by cerradão (PC)—in the city of Bela Vista de Goiás, state of Goiás, Brazil, in October 2009 and March 2010.

\begin{tabular}{|c|c|c|c|c|c|c|c|c|c|c|c|c|}
\hline \multirow{3}{*}{ FAMILY } & \multicolumn{4}{|c|}{ PP } & \multicolumn{4}{|c|}{$\mathrm{PCr}$} & \multicolumn{4}{|c|}{$\mathrm{PC}$} \\
\hline & \multicolumn{2}{|c|}{ October 2009} & \multicolumn{2}{|c|}{ March 2010} & \multicolumn{2}{|c|}{ October 2009} & \multicolumn{2}{|c|}{ March 2010} & \multicolumn{2}{|c|}{ October 2009} & \multicolumn{2}{|c|}{ March 2010} \\
\hline & $\mathrm{RC}$ & RF & $\mathrm{RC}$ & $\mathrm{RF}$ & RC & $\mathrm{RF}$ & $\mathrm{RC}$ & $\mathrm{RF}$ & $\mathrm{RC}$ & RF & $\mathrm{RC}$ & $\mathrm{RF}$ \\
\hline Species & $\%$ & $\%$ & $\%$ & $\%$ & $\%$ & $\%$ & $\%$ & $\%$ & $\%$ & $\%$ & $\%$ & $\%$ \\
\hline \multicolumn{13}{|l|}{ ANNONACEAE } \\
\hline Xylopia aromatica & & & & & & & & & 0.943 & 0.231 & 1.116 & 0.273 \\
\hline Xylopia emarginata & 0.028 & 0.185 & 0.037 & 0.195 & & & & & & & & \\
\hline \multicolumn{13}{|l|}{ APIACEAE } \\
\hline Eryngium ebracteatum & & & & & & & & & 0.582 & 0.231 & 0.039 & 0.249 \\
\hline \multicolumn{13}{|l|}{ AQUIFOLIACEAE } \\
\hline Ilex affinis & & & 1.402 & 0.195 & 1.780 & 0.264 & 1.304 & 0.356 & & & & \\
\hline \multicolumn{13}{|l|}{ ARACEAE } \\
\hline Urospatha sagittifolia & 1.545 & 7.394 & 0.581 & 4.297 & 0.703 & 0.528 & & & 1.248 & 1.157 & & \\
\hline Xanthosoma striatipes & 0.057 & 0.185 & 0.260 & 1.367 & 0.025 & 0.264 & 0.235 & 1.068 & 2.014 & 4.861 & 0.915 & 3.490 \\
\hline \multicolumn{13}{|l|}{ ARECACEAE } \\
\hline Mauritia flexuosa & 16.837 & 2.957 & 18.008 & 3.516 & 10.288 & 0.792 & 16.348 & 1.779 & 2.901 & 1.157 & 11.008 & 1.496 \\
\hline \multicolumn{13}{|l|}{ ASTERACEAE } \\
\hline $\begin{array}{l}\text { Acanthospermum } \\
\text { australe }\end{array}$ & & & & & & & & & & & 0.225 & 0.249 \\
\hline Achyrocline alata & 0.045 & 0.185 & 0.049 & 0.195 & 0.347 & 0.264 & & & & & & \\
\hline $\begin{array}{l}\text { Adenostemma } \\
\text { suffruticosum }\end{array}$ & & & & & & & 0.357 & 0.356 & 0.085 & 0.231 & & \\
\hline Ageratum conyzoides & & & & & 0.127 & 0.264 & & & & & & \\
\hline Emilia sonchifolia & & & & & 0.178 & 0.264 & & & & & & \\
\hline $\begin{array}{l}\text { Eupatorium } \\
\text { amygdalinum }\end{array}$ & & & & & & & & & 0.092 & 0.231 & & \\
\hline Eupatorium tremulum & 0.142 & 0.185 & 0.268 & 0.195 & & & & & 0.922 & 0.463 & 0.504 & 0.249 \\
\hline Mikania officinalis & & & & & 0.042 & 0.264 & & & 0.738 & 1.852 & 0.093 & 0.249 \\
\hline Praxelis kleinioides & & & & & & & 0.043 & 0.356 & & & 0.240 & 0.499 \\
\hline Vernonia ararana & & & 0.033 & 0.195 & 1.415 & 1.319 & 0.313 & 1.068 & 0.426 & 0.926 & 0.798 & 1.496 \\
\hline Vernonia helophila & & & & & 0.178 & 0.264 & & & & & & \\
\hline \multicolumn{13}{|l|}{ BURMANNIACEAE } \\
\hline Burmannia flava & & & 0.020 & 0.391 & & & 0.017 & 0.356 & 0.050 & 0.463 & 0.287 & 0.997 \\
\hline \multicolumn{13}{|l|}{ CHLORANTHACEAE } \\
\hline Hedyosmum brasiliense & & & & & & & 0.191 & 0.356 & & & & \\
\hline \multicolumn{13}{|l|}{ CYATHEACEAE } \\
\hline Cyathea atrovirens & 0.545 & 0.185 & 0.939 & 0.391 & 0.737 & 0.264 & & & & & & \\
\hline \multicolumn{13}{|l|}{ CYPERACEAE } \\
\hline Ascolepis brasiliensis & & & 0.016 & 0.391 & & & & & & & & \\
\hline Bulbostylis sellowiana & 0.106 & 0.185 & 0.833 & 2.539 & 0.703 & 0.792 & 0.070 & 0.356 & & & 0.419 & 0.499 \\
\hline Cyperus haspan & 0.293 & 1.109 & 0.199 & 0.195 & 1.364 & 2.375 & & & 0.270 & 0.463 & 0.233 & 0.499 \\
\hline Cyperus lanceolatus & & & & & 0.551 & 0.792 & & & 0.191 & 0.231 & & \\
\hline Eleocharis capillacea & & & 0.199 & 0.391 & 0.051 & 0.264 & & & 0.035 & 0.231 & & \\
\hline Eleocharis geniculata & & & & & 0.915 & 1.583 & 0.183 & 0.356 & & & & \\
\hline Eleocharis nuda & & & & & 0.034 & 0.264 & 0.339 & 0.356 & & & 0.023 & 0.249 \\
\hline Eleocharis obtusetrigona & & & & & & & & & & & 0.395 & 0.499 \\
\hline Fimbristylis autumnalis & 0.130 & 0.185 & & & 0.576 & 0.528 & 1.191 & 2.135 & 0.589 & 0.926 & & \\
\hline
\end{tabular}


Table 3. Continuation

\begin{tabular}{|c|c|c|c|c|c|c|c|c|c|c|c|c|}
\hline \multirow{3}{*}{ FAMILY } & \multicolumn{4}{|c|}{ PP } & \multicolumn{4}{|c|}{$\mathrm{PCr}$} & \multicolumn{4}{|c|}{ PC } \\
\hline & \multicolumn{2}{|c|}{ October 2009} & \multicolumn{2}{|c|}{ March 2010} & \multicolumn{2}{|c|}{ October 2009} & \multicolumn{2}{|c|}{ March 2010} & \multicolumn{2}{|c|}{ October 2009} & \multicolumn{2}{|c|}{ March 2010} \\
\hline & $\mathrm{RC}$ & $\mathrm{RF}$ & $\mathrm{RC}$ & $\mathrm{RF}$ & $\mathrm{RC}$ & $\mathrm{RF}$ & RC & $\mathrm{RF}$ & $\mathrm{RC}$ & RF & $\mathrm{RC}$ & $\mathrm{RF}$ \\
\hline Species & $\%$ & $\%$ & $\%$ & $\%$ & $\%$ & $\%$ & $\%$ & $\%$ & $\%$ & $\%$ & $\%$ & $\%$ \\
\hline Lipocarpha humboldtiana & 0.037 & 0.370 & 0.215 & 1.758 & 0.051 & 0.264 & 0.043 & 0.356 & & & 0.318 & 0.499 \\
\hline $\begin{array}{l}\text { Rhynchospora } \\
\text { consanguinea }\end{array}$ & 32.622 & 20.148 & 6.813 & 4.492 & 3.585 & 2.902 & 0.530 & 0.356 & 21.447 & 14.120 & 3.977 & 1.247 \\
\hline Rhynchospora emaciata & 0.033 & 0.185 & & & & & & & & & & \\
\hline Rhynchospora globosa & 1.923 & 1.848 & 0.573 & 1.562 & & & 0.096 & 0.356 & 1.908 & 2.546 & 5.171 & 4.488 \\
\hline Rhynchospora robusta & 2.415 & 3.327 & 1.008 & 2.344 & 11.483 & 10.026 & 4.565 & 7.117 & 1.539 & 1.157 & 2.357 & 2.493 \\
\hline Rhynchospora tenuis & & & 11.260 & 9.766 & 0.712 & 0.792 & 4.452 & 4.626 & & & 12.155 & 7.480 \\
\hline Rhynchospora velutina & 0.215 & 0.370 & & & 0.458 & 0.528 & & & & & & \\
\hline Scleria distans & 0.065 & 0.370 & 0.183 & 1.367 & & & 0.139 & 0.356 & & & 0.163 & 0.249 \\
\hline Scleria hirtella & 0.110 & 0.185 & 0.065 & 0.586 & 0.229 & 0.264 & & & 0.369 & 0.694 & 0.496 & 0.499 \\
\hline Scleria reticularis & & & & & & & 0.122 & 0.356 & & & 0.132 & 0.249 \\
\hline \multicolumn{13}{|l|}{ DENNSTAEDTIACEAE } \\
\hline Lindsaea lancea & 0.012 & 0.185 & & & & & & & & & & \\
\hline \multicolumn{13}{|l|}{ DILLENIACEAE } \\
\hline Curatella americana & & & & & 0.441 & 0.264 & & & & & & \\
\hline \multicolumn{13}{|l|}{ DROSERACEAE } \\
\hline Drosera communis & & & 0.012 & 0.195 & 0.051 & 0.264 & & & 0.050 & 0.463 & 0.116 & 0.997 \\
\hline \multicolumn{13}{|l|}{ ERIOCAULACEAE } \\
\hline Eriocaulon humboldtii & 0.451 & 1.294 & & & & & 0.183 & 0.356 & 0.865 & 1.852 & & \\
\hline Eriocaulon linearifolium & & & 0.293 & 1.367 & & & & & & & 1.202 & 2.244 \\
\hline $\begin{array}{l}\text { Syngonanthus } \\
\text { anthemidiflorus }\end{array}$ & & & 0.305 & 4.883 & & & & & & & & \\
\hline Syngonanthus appressus & 0.024 & 0.185 & & & & & & & 0.184 & 0.926 & & \\
\hline Syngonanthus caulescens & 1.240 & 5.545 & 1.085 & 3.906 & 0.271 & 0.528 & & & 0.660 & 2.083 & & \\
\hline Syngonanthus densiflorus & 0.695 & 1.479 & 1.841 & 2.539 & 0.737 & 1.055 & 0.513 & 1.423 & 1.121 & 2.546 & 3.271 & 5.236 \\
\hline Syngonanthus gracilis & 0.024 & 0.185 & & & & & 0.130 & 1.068 & 0.752 & 4.630 & 0.271 & 1.496 \\
\hline Syngonanthus nitens & & & 0.085 & 0.391 & & & & & & & 0.140 & 0.249 \\
\hline Syngonanthus schwackei & 0.122 & 0.924 & & & & & & & & & 0.070 & 0.997 \\
\hline \multicolumn{13}{|l|}{ EUPHORBIACEAE } \\
\hline Croton sclerocalyx & & & & & & & & & 0.128 & 0.231 & 0.047 & 0.249 \\
\hline \multicolumn{13}{|l|}{ FABACEAE } \\
\hline Aeschynomene paniculata & & & 0.122 & 0.195 & & & & & & & & \\
\hline Desmodium barbatum & 0.033 & 0.185 & 0.061 & 0.195 & 0.339 & 1.055 & 0.174 & 0.712 & & & 0.147 & 0.499 \\
\hline \multicolumn{13}{|l|}{ GENTIANACEAE } \\
\hline Curtia tenuifolia & & & & & & & & & & & 0.155 & 0.748 \\
\hline Irlbachia alata & 0.484 & 2.403 & 0.122 & 1.367 & 0.237 & 0.792 & 0.235 & 1.423 & 0.426 & 1.157 & 0.667 & 1.995 \\
\hline Schultesia aptera & & & 0.020 & 0.195 & & & & & 0.170 & 0.463 & & \\
\hline Schultesia gracilis & & & 0.045 & 0.391 & & & & & & & & \\
\hline \multicolumn{13}{|l|}{ IRIDACEAE } \\
\hline Sisyrinchium vaginatum & & & & & & & & & 0.099 & 0.463 & 0.085 & 0.499 \\
\hline \multicolumn{13}{|l|}{ LAMIACEAE } \\
\hline Hyptis lantanifolia & & & & & 2.737 & 5.013 & 0.557 & 1.423 & 0.206 & 0.231 & 0.388 & 0.499 \\
\hline Hyptis tenuifolia & & & 0.073 & 0.195 & 0.339 & 0.528 & & & 0.241 & 0.231 & & \\
\hline Hyptis velutina & & & & & & & & & 0.227 & 0.231 & & \\
\hline
\end{tabular}


Table 3. Continuation.

\begin{tabular}{|c|c|c|c|c|c|c|c|c|c|c|c|c|}
\hline \multirow{3}{*}{ FAMILY } & \multicolumn{4}{|c|}{ PP } & \multicolumn{4}{|c|}{$\mathrm{PCr}$} & \multicolumn{4}{|c|}{$\mathrm{PC}$} \\
\hline & \multicolumn{2}{|c|}{ October 2009} & \multicolumn{2}{|c|}{ March 2010} & \multicolumn{2}{|c|}{ October 2009} & \multicolumn{2}{|c|}{ March 2010} & \multicolumn{2}{|c|}{ October 2009} & \multicolumn{2}{|c|}{ March 2010} \\
\hline & $\mathrm{RC}$ & $\mathrm{RF}$ & $\mathrm{RC}$ & RF & $\mathrm{RC}$ & RF & $\mathrm{RC}$ & $\mathrm{RF}$ & $\mathrm{RC}$ & $\mathrm{RF}$ & $\mathrm{RC}$ & $\mathrm{RF}$ \\
\hline Species & $\%$ & $\%$ & $\%$ & $\%$ & $\%$ & $\%$ & $\%$ & $\%$ & $\%$ & $\%$ & $\%$ & $\%$ \\
\hline \multicolumn{13}{|l|}{ LENTIBULARIACEAE } \\
\hline Genlisea filiformis & & & & & & & & & & & 0.093 & 0.249 \\
\hline Genlisea repens & & & & & & & & & & & 0.279 & 0.997 \\
\hline Utricularia hispida & & & 0.033 & 0.586 & & & & & 0.106 & 0.463 & & \\
\hline Utricularia praelonga & & & & & & & & & & & 0.016 & $0.24 \mathrm{~S}$ \\
\hline Utricularia triloba & 0.354 & 0.370 & 0.370 & 1.758 & & & 0.017 & 0.356 & 0.035 & 0.463 & 0.806 & 1.496 \\
\hline Utricularia sp. & & & 0.016 & 0.586 & & & & & & & & \\
\hline \multicolumn{13}{|l|}{ LIMNOCHARITACEAE } \\
\hline Limnocharis flava & & & & & & & & & 0.461 & 0.231 & & \\
\hline \multicolumn{13}{|l|}{ LYCOPODIACEAE } \\
\hline $\begin{array}{l}\text { Lycopodiella } \\
\text { alopecuroides }\end{array}$ & 0.715 & 1.848 & 0.020 & 0.391 & 0.203 & 0.528 & & & 0.199 & 0.694 & 0.543 & 1.745 \\
\hline Lycopodiella caroliniana & 0.045 & 0.185 & & & & & & & 0.078 & 0.463 & & \\
\hline Lycopodiella cernua & 1.232 & 3.512 & 1.069 & 4.687 & 0.432 & 1.319 & 0.591 & 2.491 & 1.043 & 1.620 & 2.023 & 4.737 \\
\hline \multicolumn{13}{|l|}{ MALVACEAE } \\
\hline Waltheria tomentosa & 0.069 & 0.185 & 0.049 & 0.195 & 1.890 & 2.902 & 1.174 & 3.203 & 0.184 & 0.463 & 1.302 & 2.743 \\
\hline \multicolumn{13}{|l|}{ MELASTOMATACEAE } \\
\hline Desmoscelis villosa & & & 0.012 & 0.195 & 1.034 & 5.013 & 0.209 & 1.423 & 0.227 & 1.157 & 0.047 & 0.249 \\
\hline Macairea radula & 0.374 & 0.185 & & & & & 0.174 & 0.712 & & & & \\
\hline Miconia chamissois & 16.545 & 8.688 & 18.337 & 8.984 & 10.822 & 5.013 & 10.930 & 5.694 & 9.291 & 4.167 & 7.775 & 3.740 \\
\hline Miconia elegans & 0.394 & 0.185 & & & & & & & & & & \\
\hline Microlicia euphorbioides & 0.598 & 1.848 & 0.858 & 2.148 & & & 0.304 & 0.712 & 1.184 & 0.926 & 0.318 & 0.499 \\
\hline Microlicia helvola & 0.163 & 0.555 & 0.187 & 0.195 & & & & & 1.071 & 0.694 & & \\
\hline $\begin{array}{l}\text { Rhynchanthera } \\
\text { grandiflora }\end{array}$ & 2.695 & 3.327 & 1.130 & 2.734 & 13.797 & 12.929 & 26.261 & 16.014 & 6.121 & 6.019 & 3.597 & 4.986 \\
\hline Trembleya phlogiformis & 0.195 & 0.555 & 0.130 & 0.586 & & & & & 0.085 & 0.231 & 0.078 & 0.499 \\
\hline \multicolumn{13}{|l|}{ MELIACEAE } \\
\hline Guarea macrophylla & 0.317 & 0.370 & 1.057 & 0.391 & & & & & & & & \\
\hline \multicolumn{13}{|l|}{ OCHNACEAE } \\
\hline Sauvagesia racemosa & 0.370 & 2.033 & 0.285 & 2.734 & 0.754 & 2.902 & 0.461 & 2.847 & 0.362 & 1.389 & 0.372 & 0.997 \\
\hline \multicolumn{13}{|l|}{ ONAGRACEAE } \\
\hline Ludwigia nervosa & 1.028 & 3.142 & 0.630 & 1.953 & 1.415 & 2.902 & 0.391 & 1.423 & 3.560 & 3.935 & 1.380 & 2.244 \\
\hline \multicolumn{13}{|l|}{ PIPERACEAE } \\
\hline Piper aduncum & & & & & 1.958 & 1.319 & 1.704 & 1.068 & 0.546 & 1.157 & 1.256 & 0.748 \\
\hline Piper fuligineum & & & & & & & & & 0.099 & 0.231 & & \\
\hline \multicolumn{13}{|l|}{ PLANTAGINACEAE } \\
\hline Scoparia dulcis & & & & & 0.068 & 0.264 & & & & & & \\
\hline POACEAE & & & & & & & & & & & & \\
\hline Andropogon bicornis & 2.057 & 1.848 & 0.980 & 1.367 & 6.788 & 3.958 & 5.591 & 4.982 & 10.752 & 3.935 & 6.713 & 2.743 \\
\hline $\begin{array}{l}\text { Andropogon } \\
\text { leucostachyus }\end{array}$ & & & 0.012 & 0.195 & 0.492 & 0.264 & 0.096 & 0.356 & 0.184 & 0.231 & 0.333 & $0.24 \varsigma$ \\
\hline Andropogon virgatus & 4.850 & 5.176 & 1.016 & 3.125 & 6.932 & 4.222 & 2.922 & 3.203 & 11.326 & 5.556 & 6.426 & 4.238 \\
\hline Andropogon sp. 1 & 1.423 & 1.664 & & & 0.602 & 0.528 & 0.252 & 0.356 & 1.319 & 2.315 & & \\
\hline Andropogon sp. 2 & & & & & & & 0.130 & 0.356 & 0.603 & 0.231 & & \\
\hline
\end{tabular}


Table 3. Continuation

\begin{tabular}{|c|c|c|c|c|c|c|c|c|c|c|c|c|}
\hline \multirow{3}{*}{ FAMILY } & \multicolumn{4}{|c|}{ PP } & \multicolumn{4}{|c|}{$\mathrm{PCr}$} & \multicolumn{4}{|c|}{ PC } \\
\hline & \multicolumn{2}{|c|}{ October 2009} & \multicolumn{2}{|c|}{ March 2010} & \multicolumn{2}{|c|}{ October 2009} & \multicolumn{2}{|c|}{ March 2010} & \multicolumn{2}{|c|}{ October 2009} & \multicolumn{2}{|c|}{ March 2010} \\
\hline & $\mathrm{RC}$ & $\mathrm{RF}$ & $\mathrm{RC}$ & $\mathrm{RF}$ & $\mathrm{RC}$ & $\mathrm{RF}$ & $\mathrm{RC}$ & $\mathrm{RF}$ & $\mathrm{RC}$ & $\mathrm{RF}$ & $\mathrm{RC}$ & $\mathrm{RF}$ \\
\hline Species & $\%$ & $\%$ & $\%$ & $\%$ & $\%$ & $\%$ & $\%$ & $\%$ & $\%$ & $\%$ & $\%$ & $\%$ \\
\hline Andropogon sp. 6 & & & 0.992 & 2.344 & & & 0.357 & 1.068 & 1.972 & 2.315 & 1.287 & 1.995 \\
\hline Anthaenantia lanata & 0.317 & 0.555 & & & & & & & & & & \\
\hline Echinolaena inflexa & 0.146 & 0.370 & 0.398 & 0.391 & & & 0.104 & 0.356 & 0.177 & 0.463 & 2.829 & 1.745 \\
\hline Elionurus muticus & & & & & 0.203 & 0.264 & & & & & & \\
\hline Eragrostis sp. 1 & & & & & 0.085 & 0.792 & 0.217 & 0.712 & & & & \\
\hline Eragrostis sp. 2 & & & & & & & 0.217 & 0.712 & & & & \\
\hline Eriochrysis cayennensis & 0.707 & 0.924 & 0.224 & 0.781 & & & 0.583 & 0.712 & 0.652 & 1.157 & 0.868 & 1.745 \\
\hline Hyparrhenia bracteata & 0.695 & 0.555 & 0.081 & 0.195 & 0.144 & 0.264 & & & 1.851 & 1.157 & & \\
\hline Ichnanthus procurrens & & & & & & & & & 0.298 & 0.231 & 0.147 & 0.249 \\
\hline Luziola bahiensis & & & & & & & & & & & 0.256 & 0.249 \\
\hline Melinis minutiflora & 0.537 & 0.370 & & & 0.729 & 0.528 & & & & & & \\
\hline Otachyrium seminudum & & & 0.020 & 0.195 & & & 1.183 & 1.423 & & & 1.147 & 1.247 \\
\hline Panicum laxum & & & 0.191 & 0.391 & & & 0.643 & 1.423 & & & & \\
\hline Panicum parvifolium & 0.037 & 0.185 & 0.033 & 0.195 & 1.297 & 1.583 & 0.643 & 1.779 & 0.121 & 0.463 & & \\
\hline Saccharum angustifolium & 0.313 & 0.555 & 2.065 & 2.148 & 0.220 & 0.264 & 6.339 & 3.203 & & & 6.302 & 3.490 \\
\hline Urochloa decumbens & & & 0.167 & 0.195 & & & 0.426 & 1.068 & & & & \\
\hline \multicolumn{13}{|l|}{ POLYGALACEAE } \\
\hline Polygala leptocaulis & & & & & & & & & & & 0.039 & 0.249 \\
\hline Polygala longicaulis & & & & & & & & & & & 0.023 & 0.249 \\
\hline Polygala tenuis & & & & & & & & & 0.220 & 0.926 & 0.457 & 0.997 \\
\hline \multicolumn{13}{|l|}{ PTERIDACEAE } \\
\hline Pityrogramma calomelanos & 0.057 & 0.185 & 0.126 & 0.195 & 0.322 & 0.264 & 0.383 & 0.712 & & & & \\
\hline \multicolumn{13}{|l|}{ RUBIACEAE } \\
\hline Alibertia edulis & & & & & & & & & 0.177 & 0.231 & 0.101 & 0.249 \\
\hline Psychotria paracatuensis & 0.370 & 0.185 & 2.215 & 0.391 & & & & & & & & \\
\hline Sipanea hispida & & & & & 0.161 & 0.528 & & & & & & \\
\hline Spermacoce schumannii & 0.134 & 0.739 & 0.069 & 0.977 & 0.331 & 1.055 & 0.817 & 2.847 & 0.525 & 0.694 & 0.318 & 0.997 \\
\hline \multicolumn{13}{|l|}{ SANTALACEAE } \\
\hline Phoradendron crassifolium & 0.053 & 0.185 & 0.089 & 0.195 & 0.034 & 0.264 & & & & & & \\
\hline \multicolumn{13}{|l|}{ THELYPTERIDACEAE } \\
\hline Thelypteris biformata & & & & & & & & & 0.071 & 0.231 & & \\
\hline Thelypteris eriosora & & & & & & & & & & & 0.101 & 0.249 \\
\hline Thelypteris opposita & 0.187 & 0.185 & 0.598 & 0.586 & & & & & 0.638 & 0.463 & 0.372 & 0.499 \\
\hline Thelypteris serrata & 0.346 & 0.185 & 1.024 & 0.781 & 0.161 & 0.264 & 0.452 & 0.712 & 0.404 & 0.231 & 1.977 & 1.247 \\
\hline \multicolumn{13}{|l|}{ URTICACEAE } \\
\hline Cecropia pachystachya & 0.260 & 0.370 & 0.541 & 0.391 & 3.856 & 0.792 & 2.774 & 1.423 & 0.567 & 0.463 & 0.659 & 0.249 \\
\hline \multicolumn{13}{|l|}{ XYRIDACEAE } \\
\hline Abolboda poarchon & 0.045 & 0.185 & 0.012 & 0.195 & & & & & & & 0.186 & 0.249 \\
\hline Xyris laxifolia & 0.337 & 1.664 & 0.183 & 1.367 & 2.263 & 8.179 & 1.130 & 6.050 & 0.872 & 2.546 & 0.938 & 3.490 \\
\hline Xyris lutescens & 0.154 & 0.555 & 0.207 & 0.977 & & & & & 0.489 & 1.157 & 0.310 & 0.997 \\
\hline Xyris savanensis & 0.106 & 0.555 & 0.033 & 0.391 & 1.186 & 4.485 & 0.139 & 1.423 & 0.418 & 2.083 & 0.147 & 0.997 \\
\hline Xyris tortula & & & & & & & & & & & 0.279 & 0.748 \\
\hline Xyris sp. 1 & 1.545 & 3.882 & 0.439 & 1.758 & 0.136 & 0.792 & 0.052 & 0.356 & 0.234 & 0.926 & 0.279 & 0.499 \\
\hline Total species & 6 & & 7 & & 61 & & 58 & & 71 & & 7. & \\
\hline
\end{tabular}

Bold indicates the ten main species with the highest RC per period and per area. 


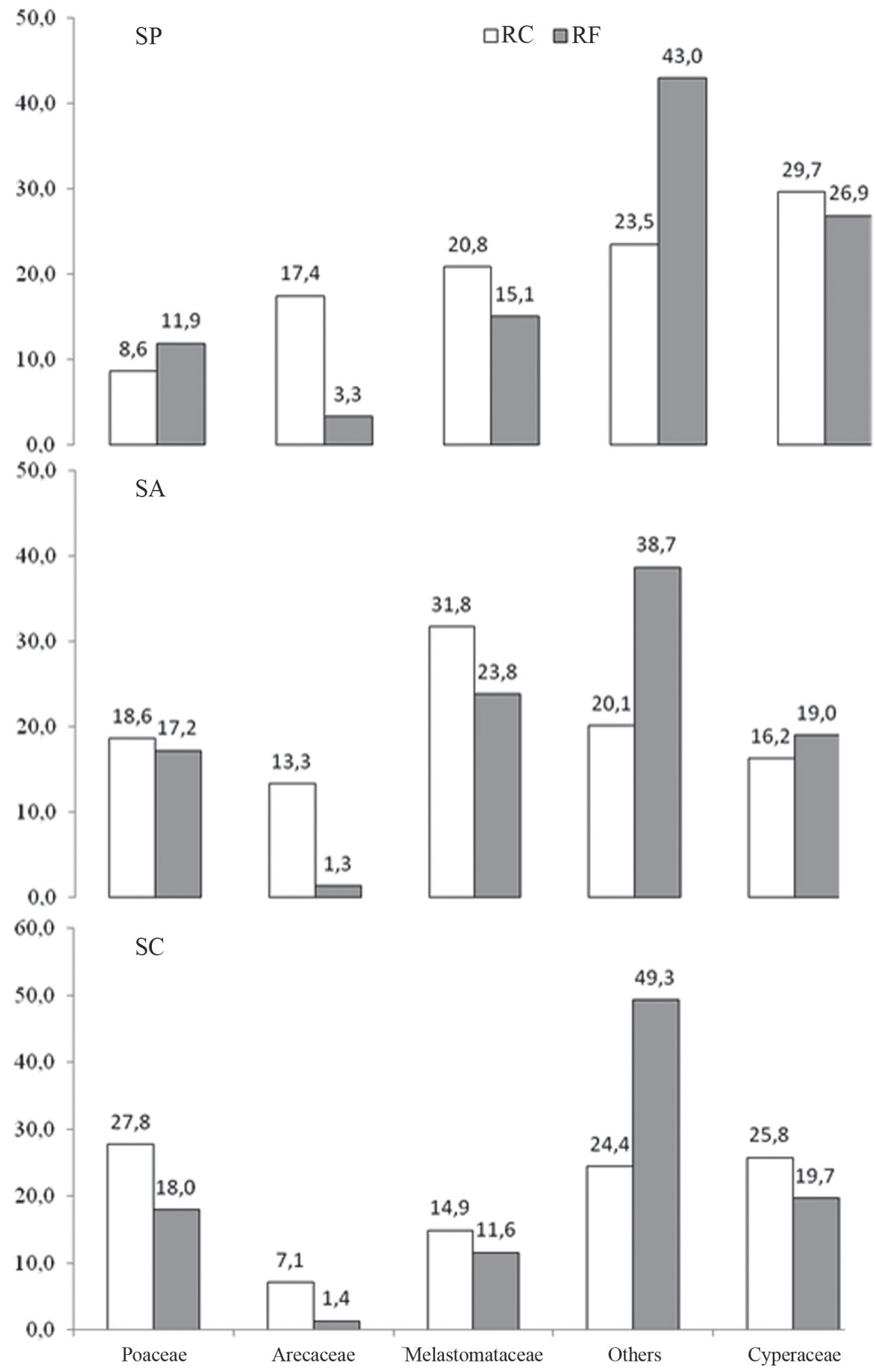

Figure 3. Distribution of cover values and relative frequencies (means of the two sampling periods), by family, for the flora of three palm swamps in the city of Bela Vista de Goiás, state of Goiás, Brazil.

PP - palm swamp surrounded by pasture; PCr - palm swamp surrounded by crops; PC - palm swamp surrounded by cerradão. 
biaceae and Fabaceae. We found that the floristic similarity was greatest between the middle and core zones within a palm swamp and lowest between the edge zones of different palm swamps. However, additional studies comparing different areas for larger periods of time are needed in order to determine whether there are patterns in the spatial and temporal distribution of plant species in palm swamps, as well as to quantify the effect that human disturbance has on these environments.

Although the herb-subshrub layer is dominant in several phytophysiognomies in the Cerrado and exhibits high species richness, as in the case of palm swamps, its vegetation has been poorly studied. This lack of data impedes the enforcement of public policies for the conservation and sustainable management of palm swamps. These environments support significant biodiversity and a rich flora. Because palm swamps occur in areas where there are natural springs, protecting these swamps also protects water resources.

\section{Acknowledgments}

This study received financial support from the Brazilian Coordenação de Aperfeiçoamento de Pessoal de Nivel Superior (CAPES, Office for the Advancement of Higher Education; doctoral scholarship to ILMR) and Conselho Nacional de Desenvolvimento Científico e Tecnológico (CNPq, National Council for Scientific and Technological Development; CTHidro Mandate Grant no. 044/2006 and research grant to LJC). We thank the anonymous reviewers for their evaluation of the manuscript. We are also grateful to the following specialists for their assistance in identifying the botanical material: Dr. Olga Yano (Bryophyta); Dr. Piero G. Delprete (Rubiaceae); Dr. Heleno Ferreira Dias (Lamiaceae); Dr. Nara Furtado de Oliveira Mota (Xyridaceae); Carlos Kreutz (Pteridophyta); Drs. Jimi Nakajima and Aristônio M. Teles (Asteraceae); Dr. Rosana Romero (Melastomataceae); and Dr. Pedro Lage Viana (Poaceae).

\section{References}

Almeida, J.R.; Baruqui, F.M.; Baruqui, A.M. \& Motta, P.E.F. 1983. Principais solos de várzeas do Estado de Minas Gerais e suas potencialidades agrícolas. Informe Agropecuário 9: 70-78.

Angiosperm Phylogeny Group. 2009. An update of the Angiosperm Phylogeny Group classification for the orders and families of flowering plants: APG III. Botanical Journal of the Linnean Society 161: 105-121.

Araujo, G.M.; Barbosa, A.A.A.; Arantes, A.A. \& Amaral, A.F. 2002. Composição florística de veredas no Município de Uberlândia, MG. Revista Brasileira de Botânica 25: 475-493.

Brasil. 1992. Resoluções CONAMA de 1984 a 1991. 4 ed. Brasília, SEMAM / IBAMA.

Cardoso, E.L.; Crispim, S.M.A.; Rodrigues, C.A.G. \& Barioni Júnior, W. 2000. Composição e Dinâmica da Biomassa aérea após a queima em savana gramíneo-lenhosa no Pantanal. Pesquisa Agropecuária Brasileira 35: 2309-2316.

Carvalho, P.G.S. 1991. As veredas e sua importância no domínio dos Cerrados. Informe Agropecuário 168: 47-54.
COPAM - Conselho Estadual de Política Ambiental. 2008. Deliberação COPAM n 367, de 15 de dezembro de 2008, MG.

Cruz, C.D. 2006. Programa Genes: Análise multivariada e simulação. Viçosa, Editora UFV.

Delprete, P.G. 2007. New combinations and new synonymies in the genus Spermacoce (Rubiaceae) for the flora of Goiás and Tocantins (Brazil) and the flora of the Guianas. Journal of the Botanical Research Institute of Texas 1(2): 1023- 1030.

Felfili, J.M.; Silva Junior, M.C.; Mendonça, R.C.; Fagg, C.W.; Filgueiras, T.S. \& Mecenas, V. 2007. Composição florística da Estação Ecológica de Águas Emendadas no Distrito Federal. Heringeriana/Jardim Botânico de Brasília 1: 25-86.

Goffinet, B. \& Buck, W.R. 2004. Systematics of the Bryophyta (Mosses): From molecules to a revised classification. Pp. 205-239. In: Goffinet, B.; Hollowell, V. \& Magill, R. (Eds.). Molecular Systematics of Bryophytes. St. Louis, Missouri Botanical Garden.

Guimarães, A.J.M; Araújo, G.M. \& Corrêa, G.F. 2002. Estrutura fitossociológica em área natural e antropizada de uma vereda em Uberlândia, MG. Acta Botanica Brasilica 16: 317-329.

He-Nigrén, X.; Juslén, A.; Ahonen, I.; Glenny, D. \& Piippo, S. 2006. Illuminating the evolutionary history of liverworts (Marchantiophyta) - towards a natural classification. Cladistics 22: 1-31.

INMET - Instituto Nacional de Meteorologia -. Gráficos e normais climatológicas. Brasília: INMET, 2009. Disponível em: <http://www. inmet.gov.br/html/clima.php >. (Acesso em 22/01/2010).

Keddy, P.A. 2000. Wetland Ecology: Principles and Conservation. Cambridge, Cambridge University Press.

Kent, M. \& Coker, P. 1992. Vegetation Description and Analysis. London, Belhaven Press.

Maltchik, L.; Bertoluci, V. D. M. \& Erba, D. A. 2003. Inventário das áreas úmidas do município de São Leopoldo, Rio Grande do Sul, Brasil. Pesquisas Botânicas 53: 79-88.

Mendonça, R.C.; Felfi li, J.M.; Walter, B.M.T.; Silva Júnior, M.C.; Rezende, A.V.; Filgueiras, T.S. \& Nogueira, P.E. 2008. Flora Vascular do Cerrado. Pp. 289-556. In: Sano, S.M. \& Almeida, S.P. (Eds). Cerrado: ambiente e flora. Planaltina, EMBRAPA-CPAC.

Meirelles, M.L.; Oliveira, R.C.; Vivaldi, L.J.; Santos, A.R. \& Correa, J.R. 2002. Espécies do estrato herbáceo e profundidade do lençol freático em áreas úmidas do cerrado. Brasília, EMBRAPA Cerrado Planaltina.

Mueller-Dombois, D. \& Ellenberg, H. 1974. Aims and methods of vegetation ecology. New York, Willey \& Sons.

Munhoz, C.B.R. \& Felfili, J.M. 2006. Fitossociologia do estrato herbáceosubarbustivo de uma área de campo sujo no Distrito Federal, Brasil. Acta Botanica Brasilica 20: 671-685.

Oliveira, G.C.; Araújo, G.M. \& Barbosa, A.A.A. 2009. Florística e zonação de espécies vegetais em veredas no Triângulo Mineiro, Brasil. Rodriguésia 60: 1077-1085.

Pereira, B.A.S.; Silva, M.A. \& Mendonça, R.C. Reserva ecológica do IBGE: ambiente e plantas vasculares. Rio de Janeiro, IBGE. Coordenação de Recursos Naturais e Estudos Ambientais.

Ramos, M.V.V. 2004. Caracterização dos solos, da estrutura fitossociológica e do estado nutricional da vegetação de veredas em diferentes superfícies geomorfológicas no Triângulo Mineiro. Tese de Doutorado. Universidade de Brasília, Brasília.

Ramos, M.V.V.; Cury, N.; Mota, P.E.F.; Vitorino, A.C.T.; Ferreira, M.N. \& Silva, M.L.N. 2006. Veredas do Triângulo Mineiro: Solos, água e uso. Ciência Agrotécnica 30: 283-293.

Rezende, J.M. 2007. Florística, fitossociologia e a influência do gradiente de umidade do solo em campos limpos úmidos no Parque Estadual do Jalapão, Tocantins. Dissertação de Mestrado - Universidade de Brasília, Brasília.

Ribeiro, J.F. \& Walter, B.M.T. 2008. As principais fitofisionomias do bioma Cerrado. Pp.151-212. In: Sano, S.M.; Almeida, S.P. de; Ribeiro, J.F. (Ed.). Cerrado: ecologia e flora. Brasília, Embrapa Informação Tecnológica: Planaltina, Embrapa Cerrados.

Salino, A. \& Almeida, T.E. 2008. Diversidade e conservação das pteridófitas na Cadeia do Espinhaço, Brasil. Megadiversidade 4: 50-70.

Schmidt, I.B.; Figueiredo, I.B.; Borghetti, F. \& Scariot, A. 2008. Produção e germinação de sementes de "capim dourado", Syngonanthus nitens (Bong.) Ruhland (Eriocaulaceae): implicações para o manejo. Acta Botanica Brasilica 22: 37-42. 
Sousa, M.A.R. de; Gomes-Klein, V.L.;Yano, O. 2010 Musgos (Bryophyta) do Parque Estadual da Serra dos Pireneus, Goiás, Brasil. Rev. Biol. Neotrop7 (1) 7-26.

Sousa, R.F. de. 2009. Atributos químicos e textura do solo em veredas conservadas e antropizadas no bioma Cerrado. Dissertação de Mestrado em Agronomia. Universidade Federal de Goiás, Goiânia.

Souza, L.F. de. 2009. Diversidade Florística e Fenologia Reprodutiva em Fitofisionomias da Reserva Pousada das Araras (Município de Serranópolis, Estado de Goiás, Planalto Central do Brasil).
Tese de Doutorado. Universidade Estadual Paulista Julio Mesquita Filho, Rio Claro.

Steinke, V.A. \& Saito, C.H. 2008. Exportação de carga poluidora para identificação de áreas úmidas sob risco ambiental na Bacia Hidrográfica da Lagoa Mirim. Sociedade \& Natureza 20 (2): 43-67.

Tryon, R.M. \& Tryon, A.F. 1982. Ferns and allied plants: with special reference to Tropical America. Springer, New York.

Yano, O. 2011.Catálogo de musgos brasileiros: literatura original, basiônimo, localidade-tipo e distribuição geográfica. São Paulo, Instituto de Botânica. 